\title{
Prolonging the Lifetime of Wireless Sensor Networks: A Review of Current Techniques
}

\author{
Felicia Engmann $\left(\mathbb{D},{ }^{1}\right.$ Ferdinand Apietu Katsriku $\left(\mathbb{D},{ }^{2}\right.$ Jamal-Deen Abdulai $\mathbb{D}{ }^{2}$, \\ Kofi Sarpong Adu-Manu, ${ }^{2}$ and Frank Kataka Banaseka ${ }^{2}$ \\ ${ }^{1}$ School of Technology, Ghana Institute of Management and Public Administration, Ghana \\ ${ }^{2}$ Department of Computer Science, University of Ghana, Ghana \\ Correspondence should be addressed to Felicia Engmann; fnaengmann@st.ug.edu.gh
}

Received 9 March 2018; Revised 10 July 2018; Accepted 17 July 2018; Published 15 August 2018

Academic Editor: Zheng Chu

Copyright (C) 2018 Felicia Engmann et al. This is an open access article distributed under the Creative Commons Attribution License, which permits unrestricted use, distribution, and reproduction in any medium, provided the original work is properly cited.

\begin{abstract}
There has been an increase in research interest in wireless sensor networks (WSNs) as a result of the potential for their widespread use in many different areas like home automation, security, environmental monitoring, and many more. Despite the successes gained, the widespread adoption of WSNs particularly in remote and inaccessible places where their use is most beneficial is hampered by the major challenge of limited energy, being in most instances battery powered. To prolong the lifetime for these energy hungry sensor nodes, energy management schemes have been proposed in the literature to keep the sensor nodes alive making the network more operational and efficient. Currently, emphasis has been placed on energy harvesting, energy transfer, and energy conservation methods as the primary means of maintaining the network lifetime. These energy management techniques are designed to balance the energy in the overall network. The current review presents the state of the art in the energy management schemes, the remaining challenges, and the open issues for future research work.
\end{abstract}

\section{Introduction}

Energy efficiency has become a major theme in wireless sensor network (WSN) research. The interest in energy efficiency may be attributed to limitations imposed by the batteries used to power such devices. These batteries are usually the main source of power for these devices and are characterized by a limited lifespan, after which they are recharged or discarded WSNs form the backbone of ubiquitous computing applications such as military surveillance, disaster, environmental, structural, health and security, and wildlife and habitat monitoring as well as precision agriculture. Deployment of sensor nodes is usually in inaccessible environments, and with limited battery capacity their lifetime is usually an issue of major concern. Several techniques have been proposed in the literature to increase the lifetime of sensor nodes as well as the sensor networks [1-6]. In recent times, long lasting sensor nodes that may never die have been proposed [7-9].

Several definitions have been proposed for the lifetime of a sensor network; however, a generally accepted definition is when the network degrades to a point when it is no longer able to perform its intended function [10]. This could be when any of the following events occur: when the first sensor node dies or when a number or percentage of the nodes die or when the network is partitioned such that there is no communication between the subnetworks or when coverage is lost [10-12].

To help extend the lifetime of sensor nodes and networks, energy conservation methods are usually employed. In this, an effort is made to reduce the energy consumed by the unit. The authors in [3] broadly categorized energy conservation schemes under the three main headings: duty cycling, data driven, and mobility driven techniques. Duty cycling is aimed at reducing idle listening when the node's radio waits in vain for frames and overhearing when nodes stay active listening to uninterested frames. Data driven techniques use some parameters of the data themselves to make decisions to reduce energy consumption during communication while mobility schemes consider the mobility of the sink or relay nodes as a factor affecting the energy consumed in the network. 


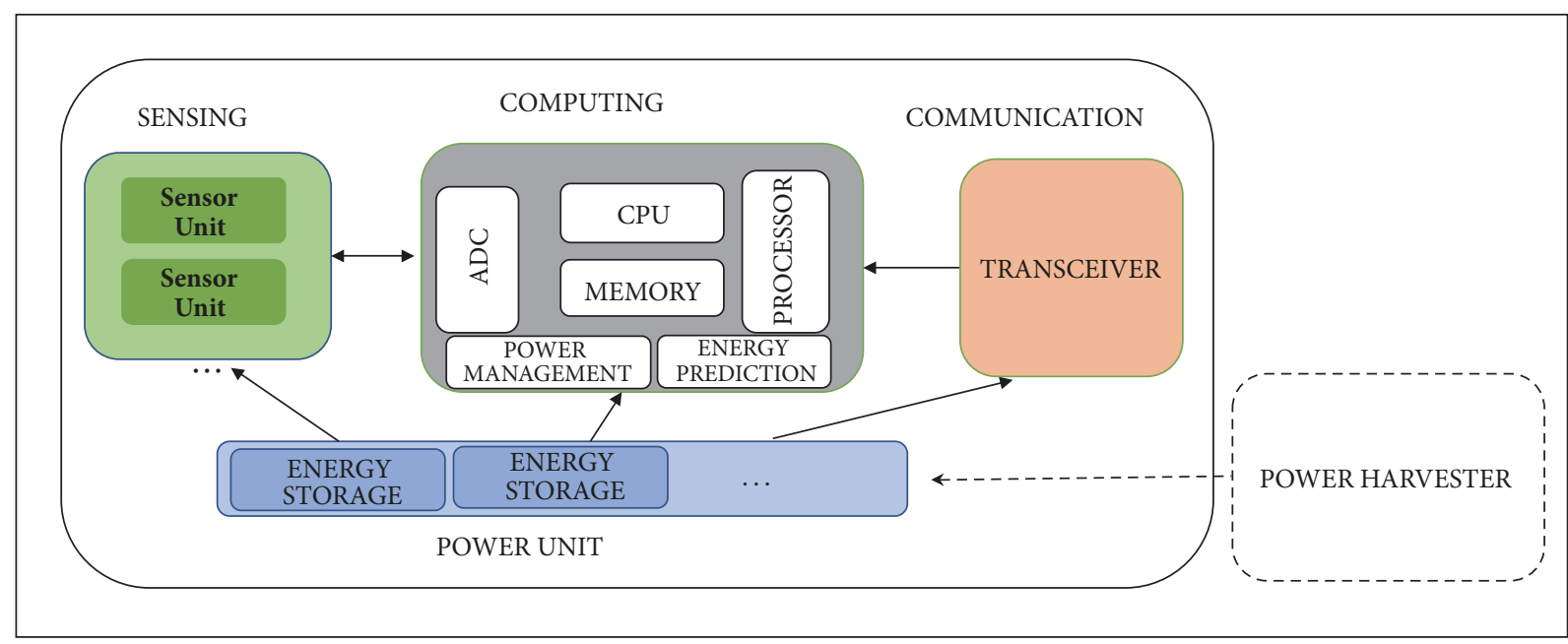

Figure 1: A typical architecture of a wireless sensor node. Adapted from [64].

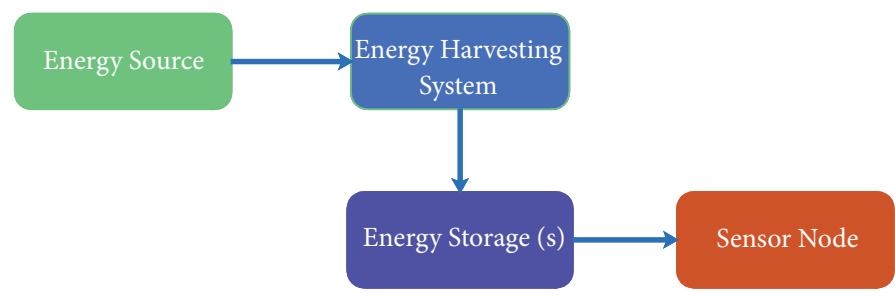

FIgURE 2: Ambient energy harvesting to store and use. Adapted from [37].

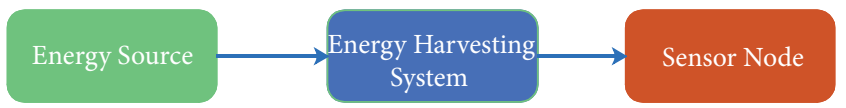

Figure 3: Ambient energy harvesting for direct use. Adapted from [37].

In Figure 1 the architecture of a typical wireless sensor node is shown. Each component of the sensor node as seen in MicaZ mote is presented. As may be observed, a typical node will consist of four major components, a sensing unit, processing unit, communications unit, and a power unit. Of the different components, the communications unit, which involves data transfer (involving both transmission and reception), expends a significantly higher proportion of the energy available [13]. This is represented in Figure 11.

Typical energy conservation techniques simply seek to prolong the lifespan of the network by reducing the energy used and do not typically require the introduction of new sources of energy. To increase the energy available to the sensor nodes, energy harvesting techniques have been proposed $[14,15]$. A key limitation of this technique is that energy sources may not always be available and hence there is the need to store the harvested energy using rechargeable batteries or low-powered supercapacitors as in Figure 2 although in some cases the energy is utilized directly by the nodes as shown in Figure 3.

Another recent technique employed for prolonging the lifetime of sensor nodes and the network is transferring energy from energy rich node to the energy deficient nodes. Energy transfer may be done wirelessly from a specialized energy harvesting node or an energy resourced node to an energy hungry node in the same network. The energy transfer may be continuous or on-demand but is limited by the cost of charge and discharge losses associated with it. Several approaches have been proposed in the literature to provide reliable energy transfer to increase the network lifetime [9, 16-22].

The method of ensuring that nodes have enough energy to function in the network by maintaining appropriate energy levels and transferring energy from an energy resourced node to an energy hungry node in the same network is referred to as energy balancing. The use of energy balancing approach to extend the life time of the networks may involve the use of any of the following schemes or a combination of them: energy conservation, energy harvesting, or wireless energy transfer. Energy conservation is the sparing use of energy in sensor nodes to allow sensor networks to be able to function as required [3]. It usually involves minimizing the communication cost in nodes $[3,15]$ since the radio is known to be the greatest consumer of the available energy $[12,23,24]$. It may also be achieved by developing energy efficient routing protocols, clustering approaches, sleep/wakeup optimization (duty cycling), and in some cases mobility $[15,25,26]$.

Energy management schemes are the techniques designed for the efficient use of energy in a network [23] and in some instances for efficient use of harvested energy 


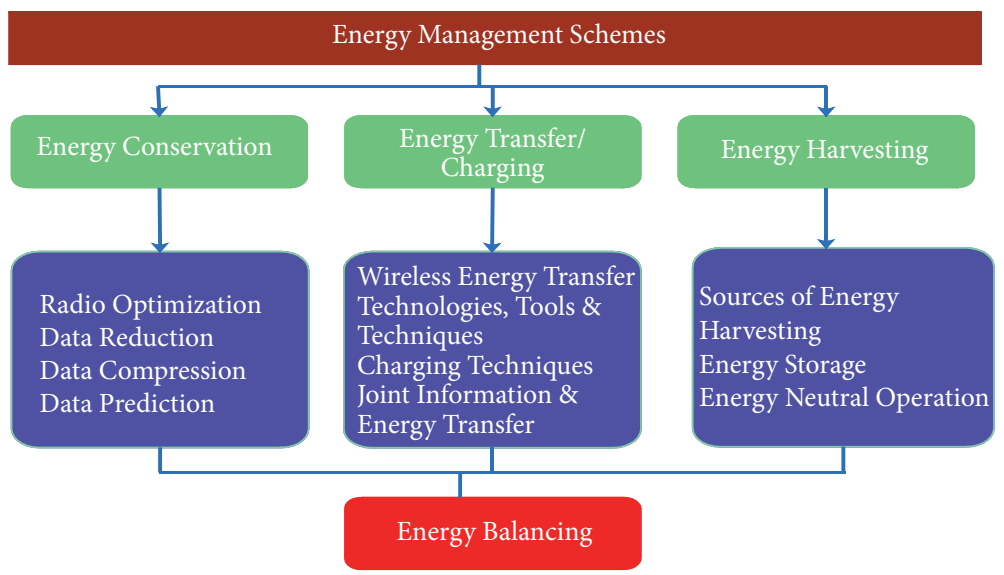

FIGURE 4: Energy management schemes in WSN.

[15]. Although some of the proposed energy management techniques assume that data acquisition through sensing consumes less energy than data transmission $[3,23]$, this may not be so for all applications [27, 28] especially in the case of energy hungry sensors, e.g., gas sensors. Most of these techniques are used to prolong the lifetime of sensor nodes by either reducing energy consumption or replenishing the consumed energy in battery powered nodes or low-powered capacitors. In this paper, we attempt to categorize the proposed energy management techniques into energy conservation mechanisms, energy harvesting, and energy transfer/wireless charging mechanisms. In some applications, [29, 30] sensing may consume significant percentage of the energy available. The broad categorizations of energy management used in this paper are energy conservation mechanisms, energy harvesting, and energy transfer/wireless charging mechanisms as presented in Figure 4. A holistic approach to achieving energy balancing in a network not only must be limited to energy harvesting and transfer but also should include energy conservation.

In this paper we present the state of the art in energy management schemes (i.e., energy harvesting, energy conservation, and energy transfer) and present the techniques used for harvesting, transferring, and conserving energy in WSNs. We discuss management schemes related to radio optimization, data reduction, aggregation, compression and prediction, and wireless transfer technologies and techniques. We present the concept of energy balancing in wireless sensor network when energy harvesting, transfer, and conservation are used efficiently. We discuss limitations in existing simulators and emulators that are designed for modeling WSN applications. Finally, we discuss the challenges and future research directions for energy management schemes.

The rest of the paper is organized as follows. In Section 2, we describe the different energy harvesting sources available and approaches. In Section 3, we describe the different wireless energy transfer techniques and technologies and present the various simulation and emulation tools for modeling recent WSNs applications. Section 4 provides current techniques for energy conservation. In Section 5 current energy balancing schemes are presented. In Section 6, we present the challenges related to energy management schemes and provide future research directions in developing wireless sensor networks that consider the trio (i.e., harvesting, transferring, and conserving) energy management scheme. Finally, Section 7 concludes the paper.

\section{Energy Harvesting}

Energy harvesting approaches scavenge for energy from the external environment such as wind, vibrations, solar, acoustic, and thermal. The techniques used in energy harvesting convert energy from the environment into electrical energy that can be used in wireless sensing nodes/devices. In wireless sensor networks, energy harvesting can be used to overcome the challenge of energy depletion that causes shorter lifetime of the nodes in the network and in other cases of the black hole problem [31]. To realize the promised benefits of energy harvesting, concerted effort is required on the part of researchers to address some outstanding issues. Energy harvesting does not guarantee immortal nodes and continuous operation due to the uncontrollable energy sources, making them unpredictable and difficult to model. The constant unavailability of energy harvesting sources is discussed in $[15,32,33]$; hence a buffer is proposed to store energy for later use, using a battery-less sensor node and low-powered capacitors to act as buffers [5, 34], as shown in Figures 2 and 3. An example is solar energy which is not available for harvesting at night due to the absence of the sun [35]. Table 1 gives specifications of some commercially available solar energy harvesting units for use in sensor nodes. In energy harvesting, nodes in the network may be attached with special devices for scavenging energy from the ambient environment for conversion into electric energy. In the case of solar energy, the size of the panel is directly proportional to the amount of energy converted through the photovoltaic technique [34]. This poses a challenge when the energy harvesting device becomes larger than the sensor node. Special energy harvesting devices may therefore be provided in the network to scavenge energy and then wirelessly transfer them to nodes. Powercast technology [36] harvests energy from intentional, anticipated, and known 


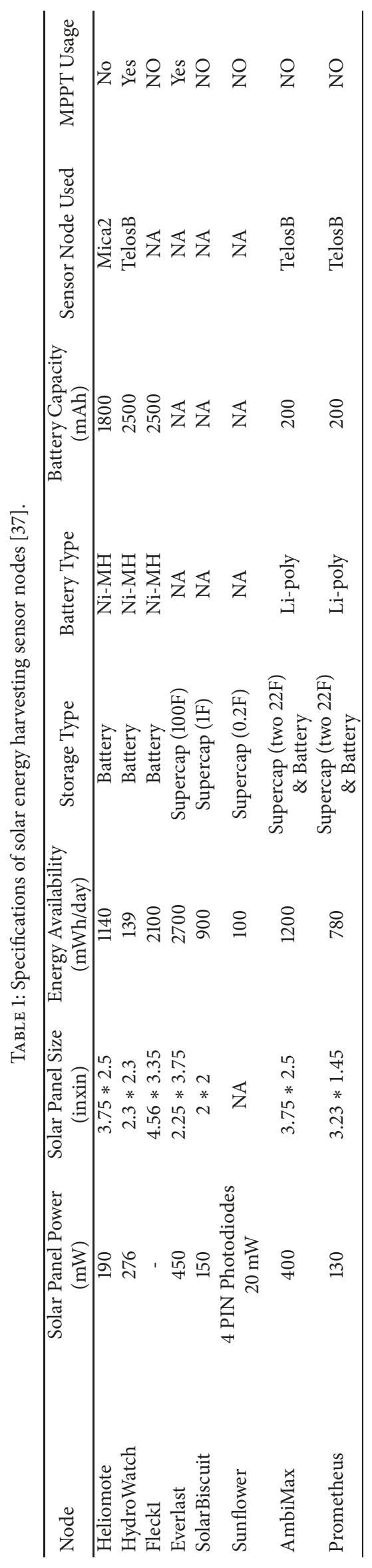


sources using the Powerharvester Receivers. Powerharvester Receivers are designed for 50 standard antennas on the 902 $928 \mathrm{MHz}$ frequency band.

2.1. Sources of Energy Harvesting. The source from which energy is harvested in a sensor network is a valuable resource since it determines the amount of energy available to the network and the rate of conversion from the source to electrical energy. Energy harvested may be classified under ambient sources, which are sources available in the surrounding environment and human sources [37]. Ambient sources of energy discussed include solar, vibration, thermal, and radio frequency.

2.2. Solar Energy Harvesting. Solar energy is an affordable and clean source of energy given its abundance in the environment. The harvesting of solar energy through photovoltaic effect is seen as the likely choice for sensor nodes with energy harvesting $[15,35,38]$. Even with its abundance there are times of the day when solar will not be available; hence there is a need for energy storage that balances the energy stored with the consumption rate of the sensor node. In [32], an energy neutral operation is employed when solar energy was the only source of energy and the sensor node has no battery. Solar energy is obtained when a solar cell receives sunlight with appropriate energy. The amount of energy derived from a typical solar system is dependent on the amount of illumination and the surface area of the solar cell with power conversion efficiencies of $15 \%$ to $25 \%$ on crystalline silicon PV cells [34]. The other known PV cells are the monocrystalline, polycrystalline, and thin-filmed based [34]. Table 1 is a summary of some commercial solar harvesting tools from [37] and their specifications making them useful in WSNs.

2.3. Vibration Energy Harvesting. Vibrational energy may be obtained through activities that produce sufficient vibrations like subways, industrial machinery, and vehicles. Amount of energy harvested is approximated in 100-W range using mechanical-to-electrical energy generators (MEEG) that use piezoelectric (ferroelectrics) and magnetostrictive materials, and electrostatic or electromagnetic mechanisms to harvest energy $[39,40]$. The harvested energy is directly proportional to the size of the MEEG used. In sensor networks where the smaller size of the node is a requirement, vibration may not be the best choice.

2.4. Thermal Energy Harvesting. Thermal energy is based on the existence of a temperature difference within an environment. The amount of energy obtainable is determined by the Carnot cycle as

$$
\frac{\left(T_{h}-T_{l}\right)}{T_{h}}=\frac{\Delta T}{T_{h}}
$$

where $T_{h}$ and $T_{l}$ are the maximum and minimum temperatures of the thermodynamic cycle.

Efficiency values up to $17 \%$ have been achieved for small temperature gradients based on the Carnot cycle [41]. Thermal energy harvesting has found application in many areas including use in devices attached to the body and implantable devices such as pacemakers for the heart. It is possible to envisage their use in other monitoring applications where a temperature difference exists. A thermal energy harvester capable of achieving an output of $100 \mu W$ was reported in [42].

2.5. Radio Frequencies Energy Harvesting. Given the large number of radio transmitters available in any urban environment, harvesting energy from this source is very appealing. Those devices capable of using harvested RF energy will have very limited power requirements. In addition, they must be in close proximity to the energy source or have a very large antenna for collecting the energy. The basic principle of operation is for the antennas to receive RF energy from the atmosphere and convert them to electrical signals as shown in Figure 5. The matching circuit is made up of capacitor and inductor components and is used to maximize RF energy in the circuit. The voltage multiplier is made up of diodes and capacitors and the resulting energy is stored in either supercapacitors or rechargeable batteries. The conversion of RF signals to DC energy is dependent on the source of the power, antenna gains, and distance between source and receiver nodes and the energy conversion rate [15], given that the power density of a receiving antenna is

$$
P=\frac{E^{2}}{Z_{o}}
$$

where $\mathrm{E}$ is the electric field and $\mathrm{Z}_{\mathrm{o}}$ is the radiation resistance of free space. Assuming $Z_{0}=377 \mathrm{ohms}$ and an $\mathrm{E}$ value of $0.5 \mathrm{~V} / \mathrm{m}$ we obtain a power density value of $0.13 \mu \mathrm{W} / \mathrm{cm}^{2}$. Electric field values larger than $1 \mathrm{~V} / \mathrm{m}$ are extremely rare. Progress in the use of RF sources will require advancement in power requirements of wireless sensor nodes.

Some of the RF technologies that exist but are not optimized for WSN use include Bluetooth, Wifi technology (IEEE 802.1la/b/h/g), and Ultra-Wideband (UWB IEEE 802.15.3). UWB has greater ratio of velocity with lower power consumption as compared to Wifi and Bluetooth but is limited to short range communications. Others that are being developed for WSN use include Wavenis by Coronis Systems, Wibree by Nokia, and Zigbee which is widely used by most WSN systems. RF power harvesting shown in Figure 5, convert RF energy emitted by RF sources such as TV signals and wireless radio networks.

2.6. Energy Storage. The use of harvested energy in WSN has a limitation since it is not always available. There is often a need to store the harvested energy for later use. Sensor nodes equipped for energy harvesting either have attached storage devices to store the harvested energy for later use as in Figure 3 or may not have storage devices but directly use the harvested energy in the node as in Figure 2. The storage devices could be either batteries (rechargeable and nonrechargeable) or supercapacitors. To replenish energy levels in WSN, rechargeable batteries and supercapacitors are used. Batteries have limited recharge cycles [5], and hence to prolong the lifetime of nodes energy conservation techniques 


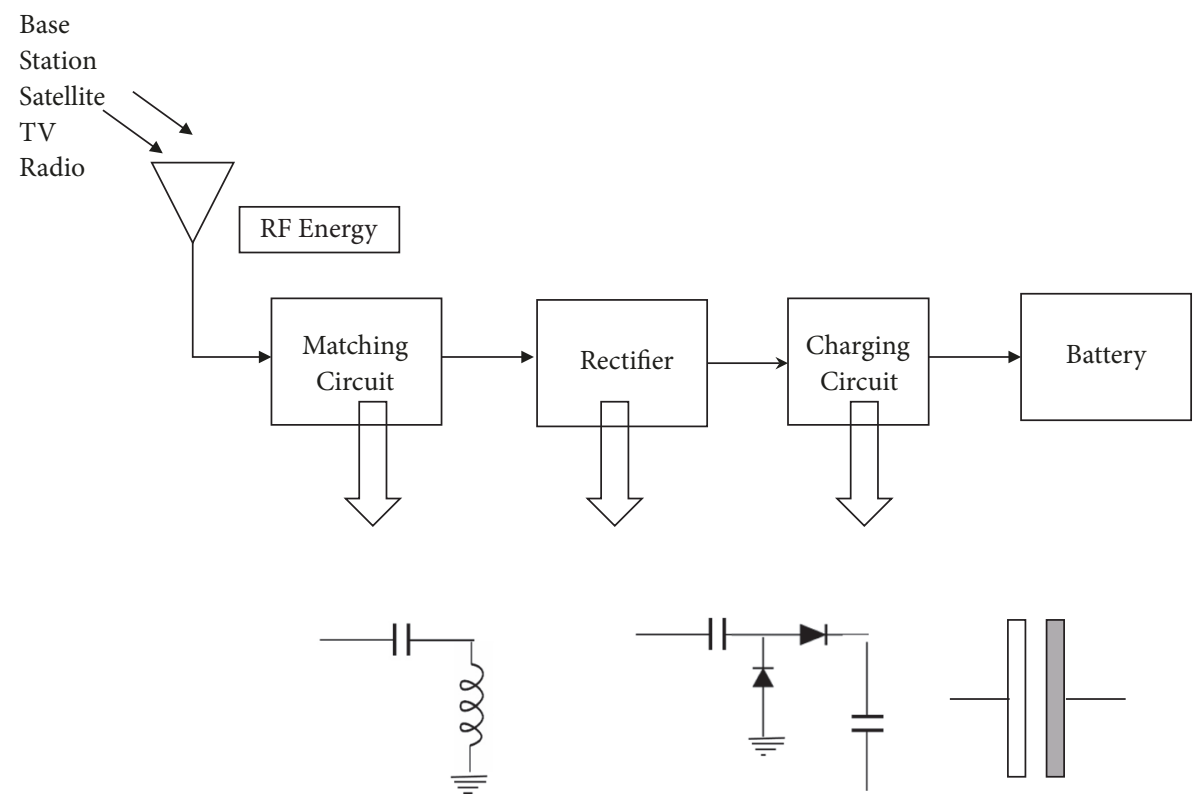

FIGURE 5: RF energy harvesting system.

must be employed together with energy harvesting. The use of supercapacitors $[5,43]$ is an alternative to batteries and may be repowered by energy harvesting. Supercapacitors may be recharged with a recharge cycle of half a million years with a 10-year functioning lifetime before the energy stored is reduced by $20 \%$ [44]. Supercapacitors have become more useful because of the high density of energy stored and their smaller size which is appropriate for WSN nodes.

2.7. Batteries. The use of batteries in wireless sensor nodes is to act as sources of energy, but with their limited capacity, energy management has become an important research area. Replacing batteries when their capacity is depleted is inconvenient in most applications of WSN. Energy harvesting gives opportunity for nodes to receive energy either from the ambient environment or from intentional sources [15]. This energy can be stored in batteries and hence the batteries must have the ability to be recharged. The amount of harvested energy is usually less than needed to charge a battery and hence must be stored to accumulate for intermittent use. Earlier power harvesting used traditional electrolytic capacitors as energy storage $[45,46]$ but with their limited energy density, their output energy per discharge cycle is very limited.

2.8. Rechargeable Batteries. Rechargeable batteries are preferred in WSN due to their high energy density [47, 48]. A $40 \mathrm{~mA}$ nickel metal hydride (NiMH) rechargeable battery could be charged from zero state to maximum state within an hour under the vibration from a typical vibrating machine. Rechargeable batteries include nickel cadmium (NiCad), $\mathrm{NiMH}$, and lithium ion/polymer (lithium) rechargeable batteries. NiCad has memory effect not suitable for shallow charging, but energy harvesting is usually slow charging. $\mathrm{NiMH}$ and lithium rechargeable batteries were discussed in
[49]. The energy densities of the NiMH are typically 60$80 \mathrm{Wh} / \mathrm{kg}$ while that of the lithium rechargeable batteries could be as high as $120-140 \mathrm{Wh} / \mathrm{kg}$. NiMH batteries are rated for 300-500 cycles while lithium batteries are rated for 500-1000 cycles, but their lifetime decreases with frequent charge/discharge cycles. Even with the higher cycle efficiency of the lithium rechargeable batteries, they are limited with shorter lifetime. The electrolyte decays causing an increase in the internal resistance. This causes the stored energy being unable to be discharged.

2.9. Capacitors. Supercapacitors are alternatives to using rechargeable batteries. Traditional electrolytic capacitors due to their low energy density are usually not encouraged in WSN. Current research proposes the use of supercapacitors. Supercapacitors are 10-100 times higher in energy density than traditional electrolytic capacitors. Supercapacitors usually have an energy density of $1-10 \mathrm{Wh} / \mathrm{kg}$ high enough for applications in WSN. They are mostly preferred in energy harvesting systems due to the higher cycles, usually rated as high as 100,000 cycles [49]. Table 1 presents some energy harvesting nodes using rechargeable batteries and supercapacitors.

2.10. Charge and Discharge Efficiency. Rechargeable and supercapacitors may not have efficiency $100 \%$. The Coulombic efficiency or charge/discharge efficiency is defined as

$$
n=\frac{\text { Discharge }_{\text {energy }}}{\text { charge }_{\text {energy }}}
$$

The types of rechargeable batteries mostly used in WSN [47, 50] are the cadmium, NiCd, nickel metal hydride (NiMH), lithium based (Li+), and sealed lead acid (SLA). SLA is not often used because even with low energy densities it also 


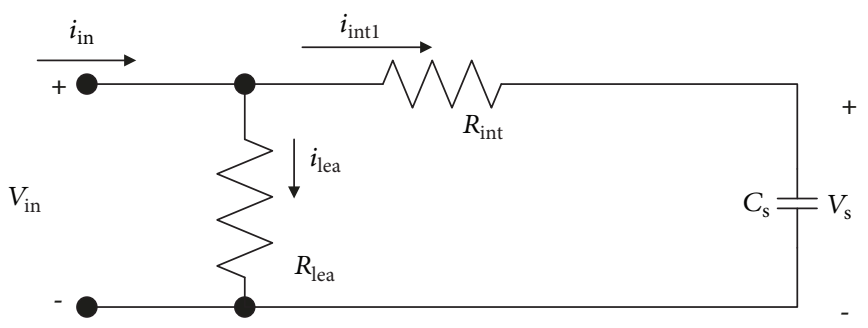

(a) Charged process

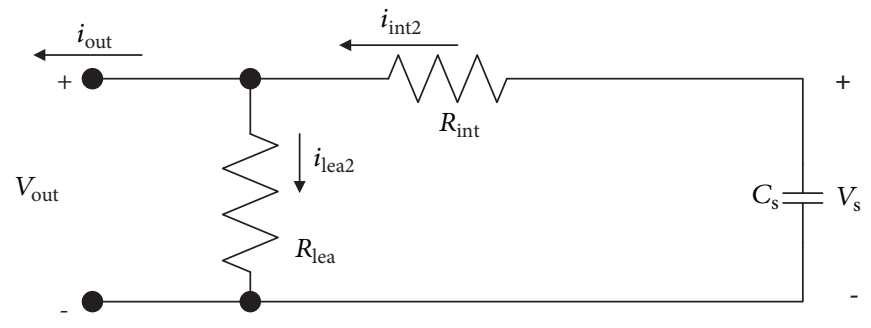

(b) Discharged process

FIGURE 6: Charge and discharge process in rechargeable batteries.

has shallow discharge cycles which causes temporary capacity losses, also known as memory effect. The $\mathrm{NiMH}$ and $\mathrm{Li}+$ batteries are mostly used, but $\mathrm{Li}+$ is more efficient. They have lower rate of discharge and longer lifetime cycle. But they are also more expensive and have complicated charging circuits, degrading faster when subjected to deep discharge cycles. NiMH batteries also degrade to $80 \%$ of their charging capacities after repeating $100 \%$ discharges, degrading to 500 cycles. Supercapacitors store charges but self-discharge at a much faster rate than batteries to as much as $5.9 \%$ on a day. They have lower energy-to-density ratios of about $5 \mathrm{Wh} / \mathrm{kg}$ with high charge and discharge efficiency of 97$98 \%$. The charge and discharge efficiencies of frequently used supercapacitors, $\mathrm{Li}+$ and NiMH capacitors, are 95,92 and $65 \%$, respectively [49]. In Figure 6 there is a diagram that shows the process of charge and discharge in a typical rechargeable battery. The NiMH rechargeable battery has the least rate of decrease and has the largest leakage loss in caparison to $\mathrm{Li}+$ and supercapacitors.

2.11. Energy Neutral Operation (ENO). In energy harvesting wireless sensor network (EH-WSN), energy neutral operation (ENO) aims at achieving the desired network performance that can be supported by the energy harvested from the required energy sources (i.e., solar, vibration, and RF) and the network-wide operations (i.e., routing, clustering, and duty cycling) over longer periods of time [32]. The implementation of WSN in environmental monitoring applications requiring uninterrupted operations has become common in recent years. In such systems, power is constantly supplied to the sensor nodes for efficient performance. The implementation of ENO generally improves the network lifetime indefinitely $[51,52]$. Several researchers have proposed different schemes to achieve energy neutrality in the network [51-53].

In [51], energy neutral operation was achieved by harvesting solar energy to improve the systems performance. The authors focused mainly on the solar available periods and designed a dynamic power management scheme that allows the system to be operational for longer periods of time. To prove the performance of their approach, trace-driven simulations were performed based on real-world data collected over 11-year period. This scheme for power management over a long-term period achieved better performance compared to other similar schemes. In [53], a real-time demonstration of vibration energy harvester was adapted to improve the network through energy neutrality. The authors measured different parameters such as data transmission and reception since these components consume a great deal of energy when the network is operational. Network-wide operations such as routing, clustering, and the node duty cycling allow wireless sensor nodes to maintain their energy levels through energy neutral operation $[52,54]$. The uncertainty in the amount of harvested energy results in a more challenging protocol design and energy prediction models for such networks. In most EH-WSN, designers and developers seek to maximize the overall network performance while meeting ENO. In order to overcome these challenges, it is relevant to compensate the energy harvesting systems by providing energy chargers and transfer power from these charges to power the sensor nodes.

\section{Wireless Energy Transfer}

Wireless energy transfer also known as wireless power transfer is the ability to transfer electrical energy from a source storage to some destination storage without any plugs or wires [55]. In 1900, Nikola Tesla experimented the wireless transfer of power from device to another without contact with large electric fields. These large electric fields diminished the energy transfer efficiency and coupled with the size of large antennas required to make these transfers feasible [8] Tesla's invention was abandoned. Due to the pervasive 
use of portable devices, wireless power transfer or wireless charging (these terms being used interchangeably in this paper) has reemerged with much acceptance, already having commercial use in applications, for example, the electric toothbrush and mobile phone wireless charging like in Apple IPhone, Samsung Qi, etc. Wireless power transfer has been achieved in applications such as RFID and medical implants using nearfield coupling. In 2007, Witricity was reintroduced by [56] who reported of powering a $60 \mathrm{~W}$ bulb from 2 meters with $40 \%$ efficiency using strongly coupled magnetic resonance [57]. Application areas include the electric vehicle charging applications [58] medical sensors, implantable devices and consumer electronics, and power transfer in concrete [59].

In [36], the transfer of RF energy (between 850 and $950 \mathrm{MHz}$, central frequency of $915 \mathrm{MHz}$ ) broadcasts radio waves in the $915 \mathrm{MHz}$ ISM band and a receiver tuning into the same frequency harvest RF power. The work [14] reports that $45 \mathrm{~mW}$ of energy is harvested within $10 \mathrm{~cm}$ of the RF transmitter with $1 \%$ efficiency. Earlier research in wireless power transfer considered transfer over single hops until [22] demonstrated the possibility of transferring energy over multihops. Their method gives room for possibility of neighboring nodes to charge energy deprived nodes that may be outside the charging range of energy charging devices like energy transmitters [22, 60]. Initial power transfer approaches had limitations in usage due to requirements such as close contact, continuous line of sight, and accurate alignment in charging direction. The work [56] experimentally demonstrated the transfer of power from magnetic inductive coil to another magnetic inductive coil that are in resonance. Resonance is achieved by the interplay between distributed inductance from a transmitting coil and the distributed capacitance from the receiving coil. Strongly coupled magnetic resonance between the coils enables the transfer of power between the coils, and this is not affected by obstructive interfaces; it is nearly omnidirectional and not limited by line of sight. The work [56] suggested that the receiving coil could be smaller for portable devices without decreasing the efficiency of transfer.

3.1. Wireless Energy Transfer Technologies. The broad categorization of wireless power transfer technologies is inductive coupling, electromagnetic radiation, and magnetic resonant coupling.

3.1.1. Inductive Coupling. Inductive coupling is the near field wireless transmission of electrical energy from a primary coil to a secondary coil. It is generated when an alternating current in a primary coil from a source generates a varying magnetic field that induces a terminal voltage of a secondary coil at a receiver. In inductive coupling, the size of coil is directly proportional to the amount of energy generated. Its charging efficiency is reduced over short distances. Its simplicity and ease of use have led to several commercial applications including electric toothbrushes, charging pads for mobile phones or laptop and medical implants and RFID tags.
The addition of a parallel capacitor to the secondary coil to form a resonant circuit at the operating frequency increases the voltage received. The Wireless Power Consortium in 2010 approved the first wireless charging standard (Qi) for low-power inductive charging. In an application where robot swarms were powered, resonance was applied on the receiving coil but not on the transmitting coil, to minimize performance variations from the interactions of the robots [61]. It is from inductive coupling that other wireless power transfer methods like resonant magnetic coupling were derived where resonance is applied to both the transmitting and receiving coils, and power transfer is done with little radiated losses. Inductive coupling operates at a frequency of 13,56 and $135 \mathrm{MHz}$. The transmission range is less than 1 meter and works best when the transmitter and receiver are in close contact $(0 \mathrm{~cm}$ giving the highest power transmission) and have accurate alignment in the charging direction. These limitations make inductive coupling not desirable in WSN.

3.1.2. Magnetic Resonant Coupling. First presented by [56], magnetic resonant coupling works on the principle of magnetic resonant coils where coils on the same resonance frequency are strongly coupled through nonradiative magnetic resonance. Energy is transferred from a source coil to a receiver coil on the same resonance frequency with little losses to external off-resonance objects. The coils could be made small enough to fit into portable devices such as sensor nodes without decreasing efficiency. Experimental results from charging a 60-W light bulb at $2 \mathrm{~m}$ in [56] reported $40 \%$ power transfer efficiency. Challenges in magnetic resonance coupling include orientation and interference, with the maximum charging distance of $2 \mathrm{~m}$ achieved only when the transmitting and receiving coils are aligned coaxially. A 45-degree rotation of the coaxial alignment reduces the coupling factor and when charging multiple devices mutual coupling between the receiving coil and other objects may cause interference. Cannon [62] demonstrated power transfer from a single resonant transmitting coil to multiple resonant receivers provided they meet these two conditions: (1) coils on the receiver must remain in the uniform magnetic field generated by the transmitting coil; (2) mutual inductances between the receiving coils must have negligible effect on the resonant interaction. This means receiving coils must be far enough from each other that their interactions with the transmitting coil are decoupled. Designing a network of mobile nodes that use magnetic resonant coupling is therefore a challenge due to the second limitation. Given the limited distances allowed in resonance coupling and the coupling interference of nodes, new research challenges are opened in power transfer in WSN.

3.1.3. Electromagnetic Radiation. Electromagnetic radiation or EM radiation emits energy from the transmitting antenna of a source to the receiving antenna through EM waves. The electromagnetic spectrum can contain regions of ambient energy levels of low and high regions and the efficiency of conversion depends on the part of the spectrum. Classifications of EM radiations are omnidirectional and 
TABLE 2: Wireless power transfer technologies [8].

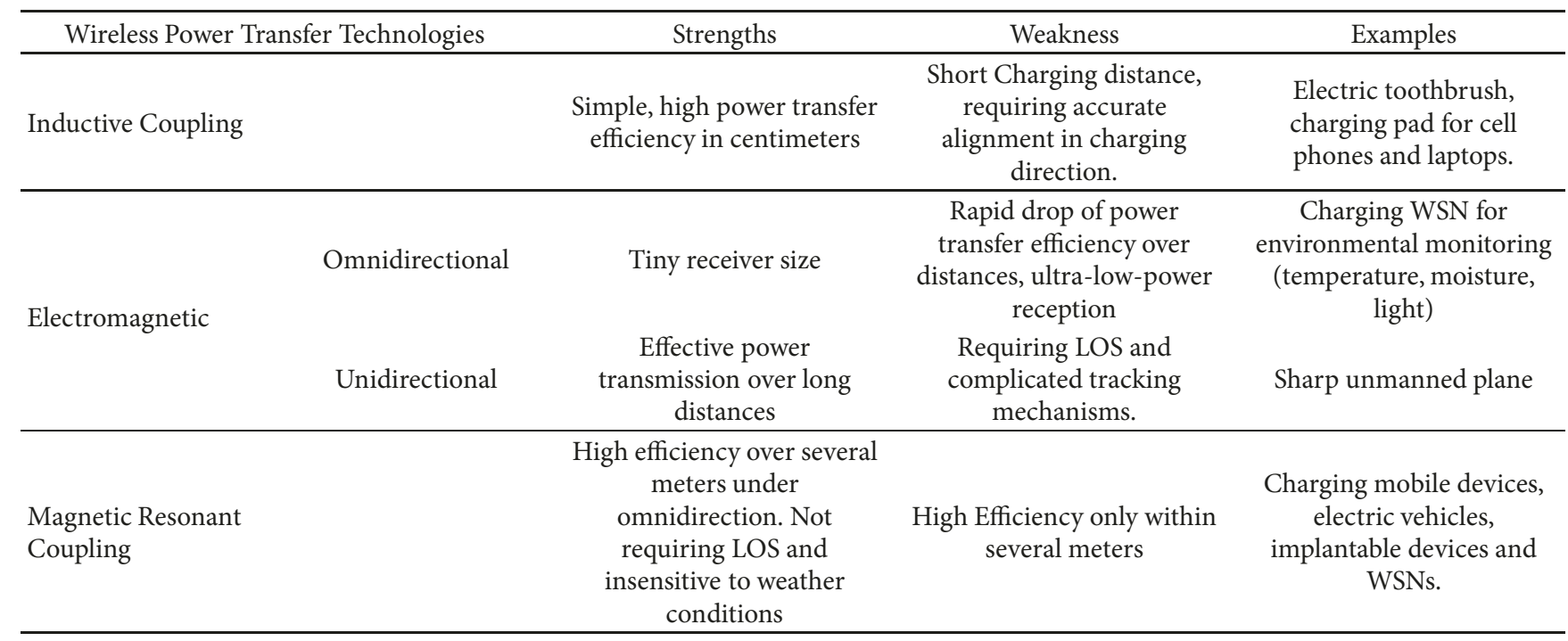

unidirectional. Omnidirectional radiation transmits broadcast EM waves in an assigned ISM band and a receiver in the same frequency harvests the radio power. Unidirectional radiation on the other hand transmits from one source to a receiving antenna in an assigned band. Omnidirectional EM waves dissipate over distances and in a paper by [8] the power transfer efficiency was $1.5 \%$ with a receiver at $30 \mathrm{~cm}$. EM radiations with omnidirectional antennas can be used in low-power sensor nodes with low sensing activities to prevent hazards to humans. To achieve high power transmission in unidirectional antennas, microwave beams transmitted on microwave frequency of 2.45 and $5.8 \mathrm{GHz}$ is used. Laserbeamed systems can be used for unidirectional power transfer under the visible or near infrared frequency spectrum. Unidirectional radiation is not suitable for wireless sensor networks because they require line of sight and has complicated tracking mechanisms. Omnidirectional radiations are used in applications where either the location of nodes is unknown a priori or nodes are mobile. Powercast technology is a commercially developed device that uses the EM waves to transfer radio frequency (RF) power from a source to receiver(s).

A summary of the wireless energy transfer technologies is presented in Table 2.

3.2. Energy Transfer Tools and Techniques. Energy transfer may be achieved using stationed energy sources that transfer energy to nodes in the network [16] or by the use of mobile chargers $[8,16,63]$. Mobile chargers have been widely used in the literature. Tools for energy transfer include mobile chargers, charging vehicles or robots, energy transmitters, and the sensor nodes themselves.

3.2.1. Mobile Chargers. The use of mobile chargers has been used by several authors when the energy of the nodes runs low $[8,69,70]$ using either human manned chargers or robots. Mobile charging vehicles have also been used $[8,55,70]$. A mobile charging vehicle carries a battery charging station that is assumed to have enough power to charge several nodes. The mobile charging vehicles may have power harvesters attached that scavenge energy from the environment and hence ensure continuous power supply to the battery station $[9,71,72]$. They could also have attached rechargeable batteries such that, after a cycle of charging nodes in the network, the mobile charging vehicles return to a stationed power source to be replenished with its energy $[55,69]$. The works $[19,20]$ studied the optimization of the vacation time of the wireless charging vehicles (WCV) over the cycle time. The use of multiple mobile chargers has also been proposed in [69] where a scheduling algorithm is used to charge sensor nodes in a large network. Single mobile chargers may not carry enough energy to recharge energy node in the network if it is not equipped with energy harvesting.

The deployment of multiple wireless chargers has been studied $[63,69,70]$ and different methods are proposed. Of such methods is triangular deployment [8] of deploying multiple mobile chargers such that a charger is placed at each vertex of the triangle. The side length of the triangle yields the minimum number of nodes for covering a plane. The method accounts for the fading of recharge signals in space unlike in binary disks. The infrastructure used even though fixed could have mobile nodes, and it is argued that having mobile nodes as opposed to stationary nodes could have the advantage of having fewer chargers. The work [73] proposed a hierarchical charging structure of multiple chargers in a network. Two groups of mobile chargers are formed: the lower mobile chargers that charge the sensor nodes in the network and the higher Special Chargers to charge the mobile chargers.

Some approaches of wireless charging include the use of specialized nodes called energy transmitters [74] to harvest energy from the environment and then transfer to ordinary nodes in the network. Electromagnetic induction, inductive coupling, and RF energy transfer techniques have been discussed and proposed in literature [14]. With all these 
approaches being discussed, there is still a need to use energy as a scares resource in the network to prolong the lifetime. Near field coupling is applied in RFID tags and medical implant. The efficiency of near field coupling techniques decays with distance at a rate of $1 / r^{6}[75]$.

3.2.2. Energy Transmitters. Energy transmitters used in research assume that the nodes have some attached antennas for receiving and transmitting energy from some device to sensor node. Powercast technology [36] that introduced the TX91501 Powercast Transmitter uses the 915-MHz ISM band to transmit radio waves for power and data. The TX91501 transmitter can broadcast power and data over 12 meters to Powercast receivers. The Powercast receivers convert RF energy into DC for devices that are batteryless or wirelessly trickle charge batteries. In [16] they realized the high broadcast range and power of the energy transmitters of as much as 3 Watts introduces interference in data communication. A model for concurrent energy and data created three regions in a network: (i) the wireless charging region within which data can be transferred directly from a transmitter to neighboring nodes; (ii) communication region where nodes can communicate with the ET but cannot be charged; and (iii) interference range where nodes receive interference from the ETs energy transmission and therefore data communication is affected during energy transfer of the ETs.

3.2.3. Monitoring Techniques. Monitoring approaches provide means to check the energy consumption levels in sensor nodes and to report the amount of energy remaining in the node. This method of monitoring energy levels gives the energy source storage nodes the ability to know which nodes to charge and the schedule for charging. Some methods and techniques employed in research for monitoring energy levels are as discussed below. In [72], three charging schemes are proposed. In two of the proposed schemes, nodes monitor their energy levels and inform mobile chargers when their energy levels go below predefined threshold. A mobile charger patrols the shortest path computed and charges nodes within the threshold. In the third scheme, a function creates a charging scheme based on the nodes residual energy and its distance to a charger for chargers that charge more than 1 sensor node.

In [71], a three-tier architecture of stationary sensors, a mobile charger, and an energy station is proposed. The energy station computes a charging sequence for the network when sensor nodes periodically send information about their energy level state to the energy station. Qi-Ferry, a mobile charger used in [76], describes the tour of the QiF as it goes through the network to charge nodes below some distance threshold. The aim of $\mathrm{QiF}$ is to maximize the number of sensors charges in a tour while reducing the amount of energy spent by the charger in a tour. Qi-Ferry monitors its energy level and iteratively removes tour stop from the tour until all nodes are sufficiently charged.

In [55] an optimal path is formulated for a mobile charger to periodically charge all nodes in the network, and this optimal path is the shortest Hamiltonian cycle. The method is to maximize the ratio of the time the mobile station spends at home recharging itself (also known as the vacation time) to the time spent in a cycle while charging nodes. The assumption is that the mobile charger is charged enough that is may not be depleted in a cycle. But this may not always be so, especially in instances where the rate of consumption of some nodes due the location-specific channel behavior of some nodes that may change. In [32], distributed energy harvesting routing is proposed where battery-less nodes using energy directly harvested from the ambient environment can predict the amount of harvested energy and based on the rate of consumption of the node, the realtime residual energy is used to create a distributed protocol. This protocol is limited and may not be practical for most energy harvesting sources whose continuous availability in the environment may not be predicted and amount harvested may not be modeled.

3.2.4. Charging Techniques. Techniques for charging sensor nodes are dependent on the source of energy being harvested and transferred and the antennas used. RF energy charging is different from the conventional constant voltage charging. This is because the RF power received for recharging a capacitor is constant for an RF source that transmits constant power to a fixed distance [65]. Some of the charging techniques for RF energy are discussed in [65] as multiple antenna transmissions, distributed beamforming of multiple antennas, and cooperative relay and protocol based optimizations. This technique is described using the illustration in Figure 7.

In [22] three charging techniques are proposed based on Witricity and investigate the possibility of transferring energy efficiently over multiple hops using long-time resonant electromagnetic states with localized slowly evanescent field patterns. The techniques are as follows:

(i) The store and forward technique assumes that nodes are equipped with rechargeable batteries. The main power source which is assumed to be stationary charges neighboring nodes till their batteries are fully charged or the source reaches an energy threshold of $50 \%$. The energy may then be transferred to nodes in the neighbors next hop and stored in batteries and then transferred to the next hop. It is assumed that nodes are attached with antennas for both transmitting and receiving energy and size and signal interference is not a problem.

(ii) The direct flow technique works on the principle that a single node can couple with multiple nodes simultaneously. Nodes couple with the previous and the next nodes in their path from the source to the destination nodes, receiving energy without storing in batteries, directly transmitting to the next node. Charge and discharge losses associated with energy transfer are not incurred except at the last node. Coupling coefficient of a pair of nodes does not affect that of the next pair of nodes since the coupling coefficient depends on the radius of the coils and not the distance between nodes.

(iii) The hybrid technique uses a combination of the store and forward technique and the virtual circuit 


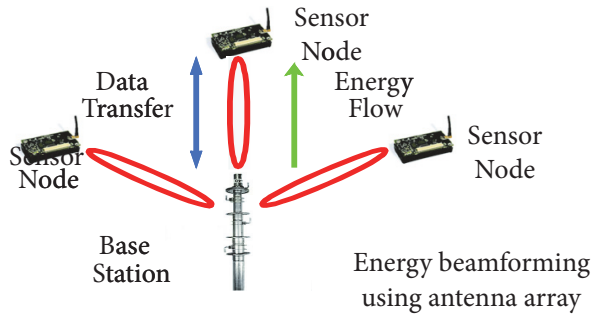

Energy beamforming
using antenna array

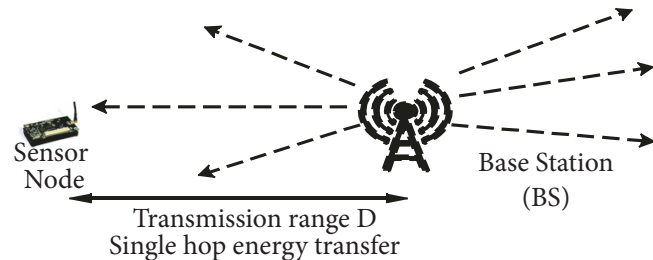

Transmission range $\mathrm{D}$
Single hop energy transfer

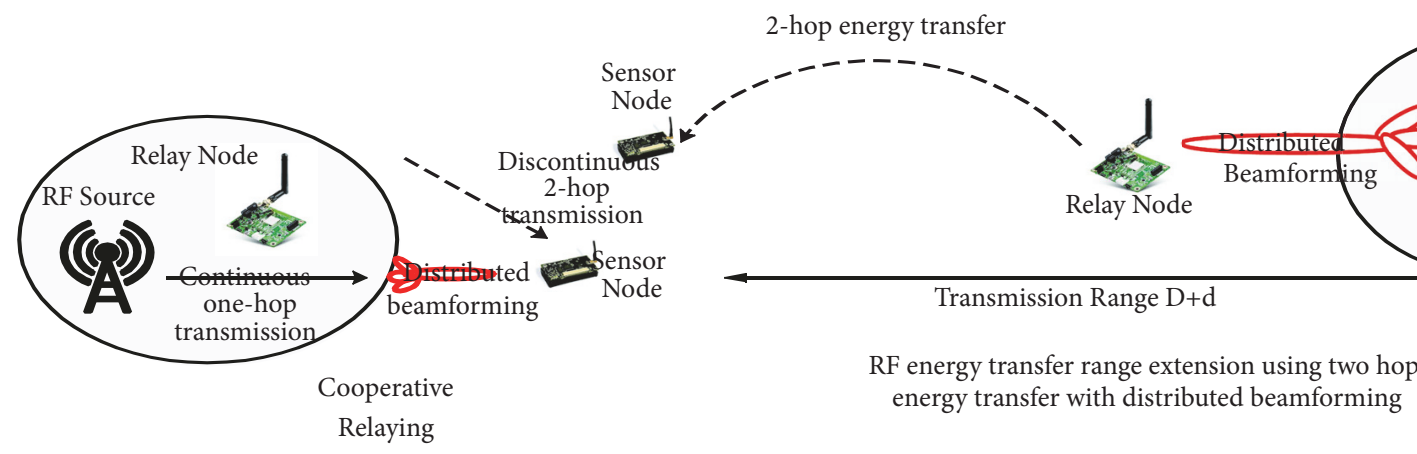

FIGURE 7: Beamforming techniques for RF energy harvesting [65].

technique. It uses a two-step approach. To transfer energy to the $\mathrm{Nth}$ node, where Mth node comes before the Nth node in terms of distance from the energy transmitter, the direct flow is used to transfer m-hops to the Mth node where it is stored; then through another direct flow technique the energy stored in the Mth node is transferred to the Nth node. The advantage is to be able to transfer to several hops while overcoming the challenges of the charge and discharge losses. The limitation of the technique that could transfer energy to as much as 20 hops is that real life test of the technique has not been made to ascertain the feasibility. Real nodes having both transmitter and receiver antennas are yet to be produced and tested.

3.3. Joint Wireless Information and Energy Transfer. RF signals carry both energy and information and with the widespread use and application in sensor networks and IoT, research into JWIET has attracted significant attention. Optimal transmission strategies and performance limitation of JWIET have been studied under perfect full channel state information at the transmitter (CSIT) [77], considering the downlink of a cellular system with single base station and multiple stations, cooperative relay system in [78], and broadcasting system in [79]. Transmission relies on the CSIT but acquisition of the full CSIT incurred large overheads [80]. Partial CSIT has been considered in $[81,82]$ with robust beamforming schemes. In [81], MISO downlink broadcasting channel with three nodes and a single MISO uplink channel in [82] are considered. In [83], a two-user MIMO interference channel in which a receiver either decodes incoming messages or harvests RF energy to operate forever is studied. A transmission strategy that achieves maximum energy beamforming and minimum leakage beamforming for a rate-energy tradeoff region is achieved. A new transmission strategy that satisfies the condition of signal-to-leakage-andenergy-ratio maximum beamforming is also proposed. A general k-user MIMO interference channel is explored in [84] where three scenarios are investigated: (i) multiple energy harvesting receiver and a single ID receiver; (ii) multiple IDs and single energy harvesting receiver; (iii) multiple IDs and multiple energy harvesting receivers, where IDs are devices for information decoding and energy harvesters are for receiving RF energy from the ambient environment. An energy beamforming scheme requires partial CSIT and reduces the feedback overhead in a two-user MIMO and kuser MIMO IFC using Geodesic information/energy beamforming strategies. A summary of beamforming schemes is presented in Figure 5. The work [65] discusses the RF harvesting efficiency prevalent in low RF-to-DC conversion efficiency and receiver sensitivity, with new communications techniques enhancing the usability of RF energy harvesting.

\subsection{Simulation and Emulation Tools}

3.4.1. Castalia Simulator. Castalia [85] is an open source simulator built on top of $\mathrm{OMNeT}++$, and it is optimized for testing distributed algorithms and protocols and features an accurate channel/radio model, radio behavior, and other aspects of communication. It has parameters for clock drift, sensor bias and node energy consumption, CPU energy consumption, memory usage, CPU time, and some implementation for MAC and routing protocols. It provides a first-order analysis of algorithms and protocols before their implementation of node platforms.

3.4.2. EKHO. EKHO [66] is an emulation tool that records and emulates energy harvesting conditions from diverse sources in the ambient environment such as solar, thermal, 

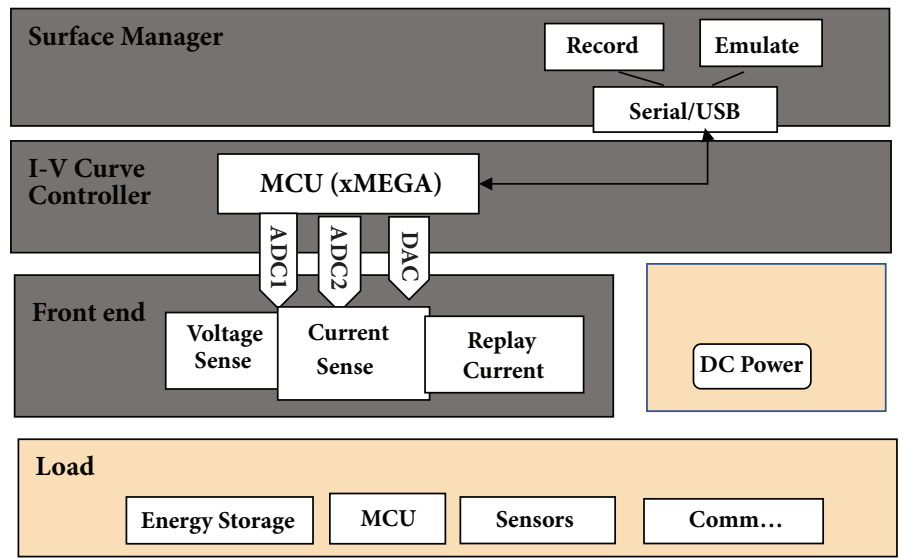

FIGURE 8: EKHO, a simulator for energy harvesting [66].

$\mathrm{RF}$, and kinetic. The energy harvesting environmental conditions are recorded and stored in a digital format which could be replayed through analog front-ends serving as energy source. EKHO is presented in Figure 8.

3.4.3. OMNeT++. OMNeT++ [86] is a discrete event simulator based on $\mathrm{C}++$ for modeling communications networks, multiprocessors, and distributed and parallel systems. It was developed as an open source tool that could be used for educational, academic, and research oriented applications, to bridge the gap between open source research oriented simulators like the NS-2 and expensive commercial simulators like the OPNET. It is available on Linux, MAC OS/X, and Windows. OMNeT provides basic machinery for users to write simulations and consists of modules that communicate by message passing. It has two major simulation model frameworks: the mobility framework and the INET framework. OMNeT++, unlike NS-2 and NS-3 which are network simulators, is a simulation platform upon which researchers could build their own simulation frameworks. It does not have a framework for energy modeling.

3.4.4. PASES. Power Aware Simulator for Embedded Systems (PASES) [67] is a SystemC based framework that is a combination of an event-driven simulation engine and a hardware, applications, and network models composer. It is a simulation and design space exploration framework that is used for power consumption analysis of WSNs application, communication, and platform layers. It gives performance and energy analysis of WSN hardware platforms and provides a gap between pure network oriented WSN tools and tools for architecture specific simulation environment. It supports Platform Based Design methodology and provides power analysis for different platforms by defining abstraction layers for the application, communication, hardware, power supply, and sensing modules of the network and node. PASES provides these abstractions: the software layer provides users with the application layer $(\mathrm{AL})$ to define application functionality.

The communication layer (CL) could be tweaked to meet network requirements such as throughput, latency, and energy efficiency. The Architecture Resource Layer (ARL) provides the service layer (SL) that provides APIs for composing different applications. The Resource Behavioral Layer defines hardware resources for target architectures and the Resource Annotation Layer specifies energy-performance details. At the energy level, the Energy Source Layer collects energy sources for sensor nodes. The Energy Source Layer provides energy source models such as batteries and supercapacitors and may include models for energy harvesting. But the energy harvesting models are not complete. PASES is presented in Figure 9.

3.4.5. COOJA. The Contiki OS Java or COOJA simulator [87] is a Contiki sensor node operating system and usually integrated with MSPSim to form the COOJA/MSPSim. COOJA allows simultaneous cross-level simulation at the application (network level), operating system, and machine code level. COOJA combines the elevated level behavior of a node to the low-level sensor node hardware in a single simulation. COOJA supports adding and using different radio mediums. It allows for the flexible additions and replacements of its parts including the radio medium, the hardware node, and plug-ins for input/output. With all the cross-level support provided in COOJA, it does not have an energy model and the energy parameters of nodes may not be properly analyzed during simulations. COOJA has a Visualizer.java class that could be extended to provide GUI and has support for memory and radio model simulations but has relatively low efficiency with increasing number of nodes hence not scalable.

3.4.6. Network Simulator 2 (NS-2). NS-2 or Network Simulator 2 is a discrete event simulator based on the Object Oriented Programming (OOP) and consists of two languages: $\mathrm{C}++$ and Object Oriented Tool Command Language (OTcL) bound together by TcLCL. Codes written in OTcL will be visualized using NAM and XGRAPH with optional python bindings. NS2 has support for protocols such as 802.11.802.16 and 802.15.4 but is limited with support for sensing. Parameters such as energy model, packet formats, and MAC protocols are different from those used in real 


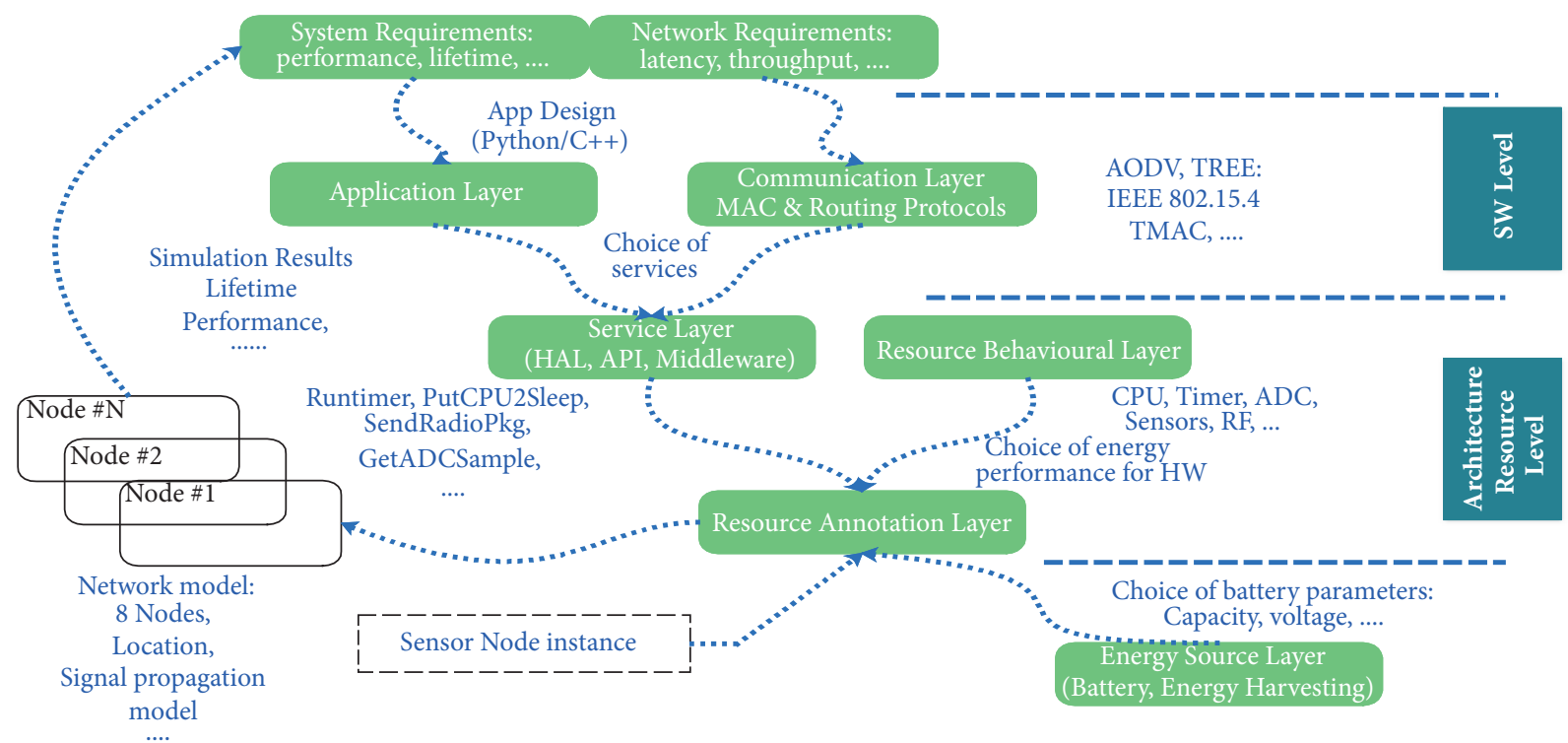

FIGURE 9: Design space exploration methodology of PASES [67].

sensor network nodes. NS-2 has parameters for residual energy but does not give models for keeping track of energy consumed by the different components and does not have a model for energy harvesting.

3.4.7. Network Simulator 3 (NS-3). NS-3 [88] is not considered an extension of NS-2 but is an entirely new simulator written in $\mathrm{C}++$ with optional python bindings. Energy model in NS-3 consists of two major components: energy source and the device energy model. The energy source is an abstract base class that provides interface for updating/recording total energy consumption on a node, keeping track of remaining energy, decreasing energy, and when the energy is completely depleted. The device energy model monitors the state of the device to calculate its energy consumption. It provides an interface for updating the residual energy in the energy source and gives notification from the energy source when energy is completely depleted and maintains a record of the total energy consumed by the device. NS-3 provides energy model for Wifi Radio with states IDLE, CCA BUSY, TX, RX, and SWITCHING. Developers may extend on the models in NS-3 to model different scenarios that may not be present in current releases.

NS-3 allows for the definition of new energy sources that incorporate the contributions of an energy harvester, with the addition of an energy harvester component with existing energy source as well as the possibility of evaluating the interaction between energy sources and the different energy harvesting models. The work [68] provided an extension of the current energy models in NS-3 introducing the concept of energy harvesting. Two energy harvesting models are as follows: the basic energy harvester, providing time-varying, uniformly distributed amount of energy and the energy harvester that recharges the energy source. A model is for a supercapacitor energy source and a device energy model is for energy consumed by a sensor node. A model for an energy predictor was introduced that is supposed to predict the amount of energy that will be available in the future based on information from the basic energy source and energy harvester. An extended diagram of NS-3 with modules for energy harvesting is presented in Figure 10.

All the above tools provide some support for energy modeling and even some simulators like the PAWiS, WSNet, OPNET, and Qualnet not mentioned above provide energy modeling but not completely. Support for energy modeling is still an open research especially when multihop energy transfer is considered in WSN.

\section{Energy Conservation}

Energy conservation methods are concerned with reducing energy consumption of the nodes. To conserve energy, the major components in a sensor node that consume energy must be controlled. The lifetime of a sensor node, which the lifetime of the network is dependent on, is an indication of how much energy is consumed and the amount of energy available for use [11].

Definition of a network frequently used in literature is of n-of-n such that

$$
T_{n}^{n}=\min T_{v}
$$

where $T_{v}$ is the lifetime of node $v$ [11]. The lifetime of the node, which is a function of energy consumed and energy available for use in the network, depends on the activities of various components, being the sensing, processing, radio, and power supply units, with typical energy consumption of the various units of the node presented in Figure 11.

The sensing component consists of the sensors with Analog-to-Digital (ADC) converters for collecting data from the environment that are then fed into the processing unit. The processing component manages the node by performing internal computations and aggregation of data with other nodes in the network and has a storage unit/memory included working as a temporary buffer. The transceiver 


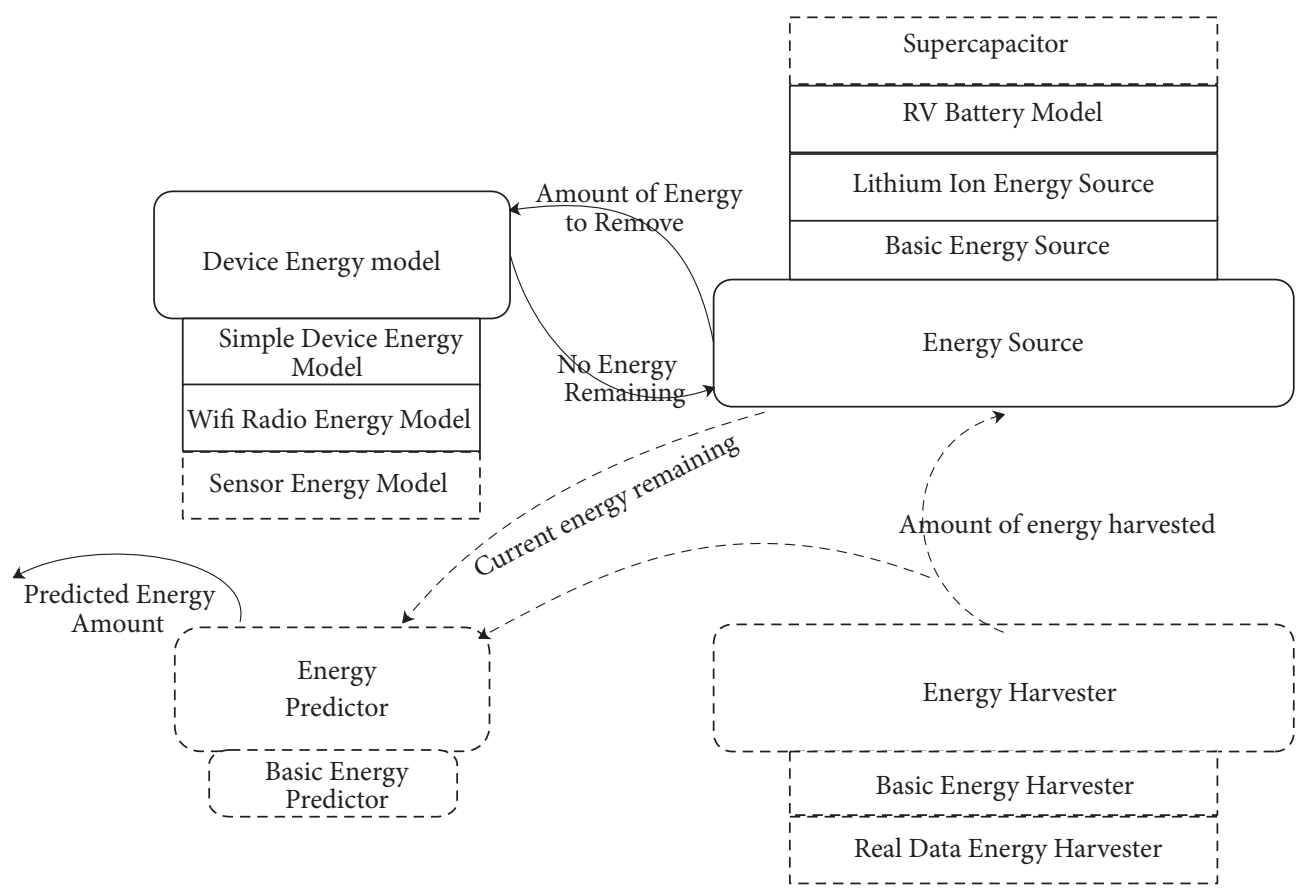

FIGURE 10: NS3 extension with energy harvesting model and basic energy model [68].

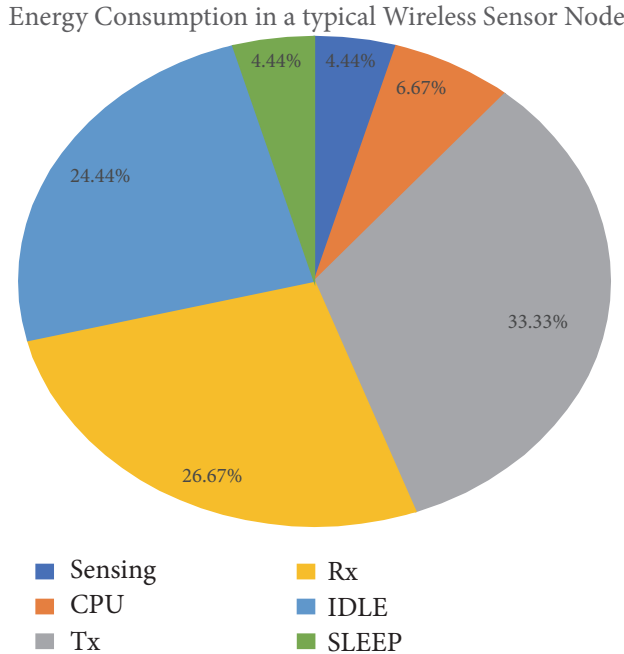

FIGURE 11: Energy consumption of the components in a sensor node.

also known as the radio unit connects the node to the network. The power unit consists of the battery or lowpowered capacitors and serves as the source of energy. They may also be supported with power scavenging units for energy harvesting. The control of the energy consumption of the various components of a sensor node has led to the generation of some methods in energy conservation which may be classified as radio optimization techniques, data reduction, and efficient routing techniques. In general, energy conservation methods focus on networking and sensing. Networking comprises the management of the sensor nodes and the design of the network protocols while sensing is based on the techniques to reduce the frequency of sensing.
Energy conservation methods provide means of reducing energy consumption by the different components of the sensor node as shown in Figure 1. Of the different components, data communication expends the maximum energy available [13], where communication involves both transmission and reception.

The sensing component consists of the sensors with Analog-to-Digital (ADC) converters for collecting data from the environment that are then fed into the processing unit. The processing component manages the node by performing internal computations and aggregation of data with other nodes in the network and has a storage unit/memory included working as a temporary buffer. The transceiver, also known as the radio unit, connects the node to the network. The power unit consists of the battery or lowpowered capacitors and serves as the source of energy. They may also be supported with power scavenging units for energy harvesting. The control of the energy consumption of the various components of a sensor node has led to the generation of some methods in energy conservation which may be classified as radio optimization techniques, data reduction, and efficient routing techniques. In general, energy conservation methods focus on networking and sensing. Networking comprises the management of the sensor nodes and the design of the network protocols while sensing is based on the techniques to reduce the frequency of sensing.

4.1. Radio Optimization Technique. The radio module is responsible for wireless communication and is the component that consumes significant amounts of energy in the network. To optimize the radio, techniques used include cooperative communication schemes, sleep/wake-up schemes, duty cycling, and radio optimization parameters such as radio 
coding and modulation techniques, power transmission, and antenna direction. The radio transceiver is one component that consumes much energy since it is used in data communication. Energy conservation methods focus more on data transmission since more energy is expended from the node during data transmission than data processing/computation. Energy consumed during sensing may be considered in energy conservation since the energy consumed may be comparable to or even greater than communication [27, $28,89]$ in some applications and cannot be ignored. Radio optimization techniques provide means of mitigating the energy consumption of sensor nodes due to wireless communication. Radio optimization techniques considered include SISO, MIMO, cooperative communication schemes, sleep wake-up schemes, and Transmission Power Control.

4.2. Single-Input Single-Output (SISO). Single-Input SingleOutput (SISO) refers to the direct transmission of data from single nodes to a base station usually through a single hop transmission. Challenges of SISO include data congestion, collisions, and loss of energy when the distance between node and base station is big.

4.3. Multiple Input Multiple Output (MIMO). Multiple Input Multiple Output (MIMO) systems assume that multiple antennas on nodes transmit data to multiple receivers. The application of MIMO spreads the power to transmit among different antennas on nodes in the network to achieve power gains. This increases the bandwidth for high data rates and bit-error-rate performance requirements [74, 90]. MIMO has challenges in WSN due to the limited physical size of typical sensor nodes that cannot support multiple antennas and the energy consumed by the circuit energy of the transmitter and receiver in the system. When the number of antennas increase, the circuit energy consumed by multiple antennas increases [74]. To mitigate the limitations of MIMO, cooperative MIMO technologies are constructed that minimize the energy consumed in transmission especially in long range transmission where the benefits of MIMO outweigh SISO $[90,91]$. SISO has efficient energy consumption for short range transmissions but still requires approaches to minimize circuit energy consumption. The reader could read papers $[74,92]$ for further benefits of MIMO.

4.4. Cooperative Communication Schemes. Cooperative communication schemes provide means of communication by allowing the terminals/antennas in a multiuser environment to collaborate in communicating in a sensor network, using the broadcast nature of wireless networks. Single antennas in a multiple user environment collaborate to share their antennas to form a virtual multiple antenna transmission, thereby gaining the benefits of MIMO systems while overcoming their challenges, such as size, cost, hardware, and deployment limitations [76, 93]. Wireless nodes in cooperative communication systems act as transmitters and also cooperative agents for other users. Two ways of cooperation are introduced in [76] called the relay cooperation and node cooperation. Relay cooperation is when extra relay nodes help to transmit data from source to destination. This is achieved by relay nodes receiving data from sources and then transmitting to destinations using some cooperation protocols (amplify-and-forward, decode-and-forward, and compress-and-forward). In node cooperative systems, nodes cooperate by either communication terminal using combined processing or coordinating the strategies for communication at the terminals. Challenges in the implementation of cooperative communication systems include, but are not limited to, cooperation assignment and hand-off, network interference, transmitting and receiving requirements of wireless systems, and the loss of rate to the cooperating mobile system [93].

4.5. Sleep/Wake-Up Schemes. Sleep/wake-up schemes adapt the node to the activities of the network to conserve energy by putting the radio to sleep, to minimize idle listening (idle sensing of the channel). Duty cycle is defined as the ratio of time nodes that are active during their lifetime and the sum of the times when the node is on and asleep [94], which means nodes alternate between sleep and wake-up times. The radio transceiver of nodes is made to sleep when there is no communication and wakes up when data transmission is required. The alternating of sleep and wake-up periods is referred to as duty cycling. The downside of this technique is that data generated during the vacation period (when node is sleeping) may be lost. Another technique that is data driven senses the channel until some data is generated; then it wakes up for transmission. Unnecessary data may be transmitted to the sink increasing energy consumption and could also be too energy consuming if the data sensing is not negligible.

To optimize the sleep period, event-driven systems adapt selective and incremental wake-up scheme, where lowpowered sensors continuously monitor the system, until some event trigger is received; then nodes are triggered for high-quality detection and quality sensing. Another method puts all nodes to sleep but they wake up when there is a demand by another node to communicate. This means nodes are active only for a minimum time during communication. No sensing of the network is required and it is appropriate for applications where sensing consumes much energy and periods of data communication are known a priori. Thirdly, there are methods where all nodes sleep and wake up at the same time according to a wake-up schedule. These methods are appropriate for data gathering applications where aggregation may be required but more collisions will be introduced in such networks as all nodes wake up at the same time. Asynchronous methods allow nodes to wake-up independently with overlapping wake-up periods with the neighbors. Such networks require active periods of sensing or nodes will have to wake up frequently when sender sends long preamble or receiver remains active for longer periods. All will require huge energy cost to the network and hence this is not an efficient energy conservation technique, but good for QoS purposes [3].

4.6. Transmission Power Control. The aim of Transmission Power Control (TPC) approaches is to dynamically adjust the transmission power of the radio to maintain an effective communication link between pairs of nodes while not transmitting at full power capacity $[95,96]$. Factors such 
as distance and link quality affect the transmission power within a transmitter-receiver pair. A survey of TPCs by [95] investigated existing approaches of protocol development that were based on single-hop communication in WSN. An Adaptive Transmission Power Control (ATPC) [96] was proposed that builds a model for communication where neighboring nodes create a correlation between transmission power and link quality. A feedback-based TPC algorithm is employed to dynamically maintain individual link quality, creating a pairwise adjustment for ATPC that saves energy with online control and is robust to environmental changes. A TPC method for SCADA systems is used for industrial control of their remote stations and a central site [97]. Using a fuzzy based algorithm, a minimum number of transmission paths are maintained between the sink and source nodes while maintaining minimum multihops. The effect of different Transmission Power Control protocols on the lifetime of WSN is studied in [98] when power levels and strategies for transmission power assignment are discretized. The bandwidth of TPCs and the granularity of the power control of the link-level affect the energy consumed.

4.7. Data Reduction. Data reduction techniques in WSN reduce the amount of data that is transmitted to the destination, usually the sink, thereby reducing the number of transmissions. These techniques reduce the bandwidth needed to send data as it traverses the network from source to destination (usually the sink). Some techniques used are data aggregation, compression, and prediction. Others are network coding and efficient routing.

4.8. Aggregation. Aggregation techniques fuse data as it traverses the network from one node to the other to the sink. Its main aim is to aggregate data in an efficient manner to increase the network lifetime [99]. Since near nodes share similar data by spatial correlation, energy is wasted when the same data value is routed from multiple sources to the sink. Transmitting $1 \mathrm{~KB}$ of data over $100 \mathrm{~m}$ expends energy as much as executing 300 million instructions on a typical processor with 100MIPS [100]. In-network data aggregation can be broadly categorized as Address-Centric (AC) and Data Centric (DC). It reduces medium access contention and the number of transmitted packets and minimizes packet transmission delays. Aggregation in the network can be done via data aggregation tree (DAT for flat networks) and by clustering for hierarchical networks. Some key points in data aggregation are as follows:

(i) Nodes sense data values on the entire network and route to neighbor nodes.

(ii) Sensor nodes can receive different versions of the same message from different nodes in the network.

(iii) Data is combined from diverse sources and routes to mitigate redundancy.

(iv) Intermediate nodes must access the content of packets to be aggregated.

(v) Nodes must wait for a predefined waiting time (WT).
A survey of data aggregation algorithms is presented in [101] and analyzes different solutions against performance metrics such as data latency and accuracy. In data aggregation algorithms, there is usually latency and accuracy based on the application area. Energy efficiency, aggregation freshness, and collision avoidance are some performance metrics used in data aggregation.

4.9. Data Compression. These are techniques that reduce the size of sensed data before transmission. This reduces the amount of energy consumed in processing and transmitting data in individual nodes in the network, reducing the size of bandwidth used. A basic assumption in compression is that the amount of energy consumed compressing $a$ bit of data into $b$, such that $a<b$, must be smaller than the amount consumed in transmitting $a-b$ string of data [102]. The work [103] presented a survey of mechanisms for data compression. The assumption used in data compression is that multiples of energy consumed per 480 addition instructions are consumed for every bit of data transmitted by radio. If more than 1 bit of data is taken from sensed data by data compression, total power consumed by transmitting that data will be significantly reduced. Data compression techniques in WSN take into consideration the size of the compression algorithm and the processing speed (that of a typical WSN node is $128 \mathrm{MB}$ and $4 \mathrm{MHz}$, respectively). Examples of these compression techniques are coding by ordering, pipelined in-network compression, low-complexity video compression, and distributed compression [102].

4.10. Data Prediction. Prediction is a term given to the process of inferring missing values in a dataset based on statistical or empirical probability or the estimation of future values on some historical data. A prediction method is a function with two inputs, the set of observed values and the set of parameters. A model created for prediction is deterministic and obtained from the observed values, but one could have several prediction models from the same prediction method or algorithm [104].

Prediction methods require additional information about the observed data which may be known to the user before deployment which can be applied to the statistical data. The additional information may support assumptions made in predictions that determine the feasibility of the model. This feature of prediction models makes them more reliable compared to machine learning techniques that use fewer assumptions of the data in exchange for the time to adjust parameters and adapt to the observed data set. This does not give the user the opportunity to see the prediction accuracy before testing with real data. The work [104] groups prediction schemes under two main headings: single prediction schemes and dual prediction schemes. Single prediction schemes are made at one point in the network which could either be closer to the sensor nodes or close to the data collection point. In this, sensor nodes may sense data but based on the reliability of predictions of the sensed data predict changes to the amount of data measured and transmitted. The advantage is that each device may decide to adapt itself based on the predictions or not without a 
need to synchronize with neighbors of their decisions without incurring any overhead cost of communications. This could eventually reduce the quality of the information derived from the cluster heads since most data may not be transmitted from the sensor nodes. With the autonomy of cluster heads coupled with the spatiotemporal correlation of sensor nodes placed near each other, probabilistic models could be generated with good distributions and confidence levels that may be used to predict measurements thereby reducing transmissions.

Applications of this models are used in adaptive sampling $[105,106]$, clustering [107], and data compression [108, 109]. Dual prediction schemes on the other hand make predictions of the cluster heads and in sensor nodes. When sensor nodes measure values outside the threshold of the prediction models, the value from the sensor nodes is transmitted to the cluster heads which then sends to the sensor nodes the correct value. Frequent transmissions and therefore energy consumption are hereby reduced. The aim of dual prediction schemes is to mitigate the number of transmissions without compromising on the quality of measurements made by the systems and hence a tradeoff between the number of transmissions for new prediction model distribution and the reliability of the channel is always made $[109,110]$.

\section{Energy Balancing}

Energy balancing techniques that distribute the amount of energy in the network such that nodes have equal amount of energy and have prolonged lifetime have been discussed in this paper. They comprise data reduction schemes that reduce the amount of data that is delivered to the sink node and balancing schemes that optimize the distribution of energy available to the node and energy consumption of the nodes in the network.

Balancing schemes proposed in recent research discussed means of distributing and managing the energy in a sensor node [23]. Clustering schemes have been used to balance energy in the network, and they were processes where nodes are grouped together with a coordinator, usually known as the cluster head, that perform specialized functions such as data fusion and aggregation, and communicate this data from its clusters to the base station. Most published clustering approaches form groups of nodes and allow these nodes to select a cluster head based on some criteria. The cluster head selection can be randomized [111] or based on degree of connectivity [1]. Some approaches include the residual energy of the node as a criterion for cluster head selection [7, 112].

The possibility of unbalanced energy consumption in the network was due to the different consumption rates of energy of nodes and their distance from the base station, causing some clusters to be of high energy while others are of low energy, in a situation known as the black hole problem [31]. To solve this problem, the unequal clustering approaches [113] have been proposed. A round-robin method causes cluster heads to be rotated among the nodes in the network of homogeneous nodes (beginning the network formation with the same energy level) and have the same capabilities. The assumption is that due to different transmission and reception rates of data of the individual nodes energy depletion will not be the same throughout the network. Rotating cluster heads changes the topology of the network at each round of rotation and imposes change over overheads [114, 115].

All cluster heads in the network must be notified of the change while cluster heads change their routing tables and scheduling strategy. Some methods include the addition of high energy specialized nodes in the network to balance the load in various locations of the sink node to balance energy consumption. In [116], high energy nodes called gateways are proposed which form equal sizes of clusters in the network based on the cost of communication and the load on the gateways. These gateways act as cluster heads and perform energy consuming tasks like data fusion and organization of nodes for special tasks. The method solves the problem of extra overhead incurred by frequent reclustering on nodes since this task is performed by the gateways. The addition of specialized nodes comes at an extra cost and the optimized number added in a network must be considered. Other methods balance the energy consumption in the network by forming clusters of unequal sizes [7, 117, 118]. In these approaches, the size of clusters increases as one approaches the base station. The assumption is that, for nodes further away from the base station, multihoping data through relay cluster is more energy efficient than directly since the amount of energy required to transmit data from one node to another is directly proportional to the distance between the two nodes. Cluster heads aggregate data from their clusters and relay data from other cluster heads to the base station. This means cluster heads closer to the base station will be depleted of their energy faster than nodes on the peripheral.

This paper includes energy harvesting schemes augmented with energy transfer technologies to distribute energy available in the network fairly such that nodes are not depleted of their energy below some threshold when they are no more operational. Energy conservation techniques are also included to ensure efficient use of energy by the sensor node with minimal consumption.

\section{Challenges and Future Research Directions}

In energy transfer in wireless sensor networks, some researchers have attempted to solve the distance related energy transfer issues $[8,34,55,63]$. Despite the attempts to resolve these issues, there remains a great deal of work in this area. In this section, we present some challenges in energy harvesting and energy transfer and propose likely future works.

\subsection{Challenges}

Cost of Experimentation Using Testbeds. Research in WSN requires comprehensive evaluations process that could be verified and reproduced. Most studies done over the years have evaluated theoretical analysis and simulations lacking experimental evaluations. Over the years research into the transfer of energy from node to node or from an energy transmitter to nodes by either single hop or multihop has been proposed as a solution to making sensor networks immortal. Most of such research is based on the modeling of the 
networks and the charging scenarios. Creating real testbed experiments for energy transfer is still ongoing research with a little breakthrough. The work [56] performed simulation and temporary transfer of energy over magnetically coupled resonance coils of $1 \mathrm{~m}$ diameter or less with $60 \%$ efficiency. This shows a direct transfer without storing or retransmission of the power. Powercast Technology [36] has the Powercast energy transmitter that provides an EIRP of $3 \mathrm{~W}$ for $5 \mathrm{~W}$ DC input but does not generate continuous RF output. The work [22] proposed a solution for multihop energy transfer using theory and simulations to investigate the phenomenon of slowly evanescent field patterns that can transfer energy efficiently. Their results proved the transfer of energy over 20 hops but lack testbed tests. The work [119] demonstrated multihop RF energy transfer within two hops using the MICA2 mote operating on the supercapacitors on the Powercast P1110 Evaluation Board. The setup was made of A HAMEG RF synthesizer HM8135, an intermediate node which is made up of the P1110 Evaluation Board, and a modified MICA2 mote powered from the $50 \mathrm{mF}$ supercapacitor on the board and has a $6.1 \mathrm{dBi}$ antenna for transmitting energy in the form of data packets to the end node (P1110 Evaluation Board). This setup is not automated and requires reconfigurations each time the nodes position or topology changes. It is also not scalable and is limited to two hops with modified sensor nodes. For efficient energy transfer, cross-layer support for energy transfer comprising the MAC, link, and application layers is critical for the implementation of wireless energy transfer in WSN. There is lack of hardware designed to support energy transfer and the lack of optimal energy-aware routing protocols that consider the concurrent transfer of energy and data in a network.

For charging nodes in an entire network, specialized devices such as mobile chargers or robots have been designed, with shortest path algorithms and optimal paths developed for easy charging, but these specific nodes increase the overall cost of energy transfer on test beds. The introduction of specialized nodes for energy harvesting and transfer called energy transmitters also increases the cost of implementation. Sensor nodes due to their usage and places of deployment are expected to be smaller in size; this becomes a challenge when antennas for energy transmission and reception must both be attached to common nodes for receiving and transmitting energy.

Designing Energy Transfer Models in Current Simulators. Simulation tools of wireless sensor networks currently lack features that support useful energy models for energy harvesting from renewable and sustainable energy sources [34]. There is a need to either develop energy models in existing simulation tools for energy harvesting and monitoring or develop energy modeling simulators for wireless sensor networks. NS-2 energy model comprises the radio energy model parameters and allows a user to set the initial energy on the mote but does not have models for energy harvesting or the ability to transfer energy from node to node. NS3 has an energy model which has models for the device energy model, the fundamental energy source (which is usually the Li-ion battery but can allow for modifications with other energy sources like supercapacitors) and the energy harvesting model. The energy harvesting model increases the energy stored in the energy source and could be modeled as some other energy harvesting source [68] like in solar energy harvesters in [120]. Other simulators like Castalia, EKHO, and COOJA do not have models for energy modeling. Castalia does not provide battery or energy modeling and therefore does not support lifetime estimation simulations. It also does not have postprocessing tools for GUI support [86]. PASES [67] is a design space framework that was created with power awareness for the different components of the sensor node. It has the energy level that introduces the Energy Source Layer that analyzes the power consumption of the hardware components and models for energy sources like batteries and supercapacitors and energy harvesters. It has model for the device energy model but does not give a user the flexibility to customize protocols at the MAC and network layer; files are in XML and Python and do not support other low-level languages like $\mathrm{C}$ and $\mathrm{C}++$ which is used in most simulators and test beds. Interfacing PASES with testbed tools like what is done in COOJA is not possible and PASES does not also support increasing number of sensor nodes being added at runtime.

Prediction Models for Energy Harvesting. The unpredictability of energy due to the continuous supply of energy harvesting sources to predictive models for energy harvesting that depend on the residual of energy in the network based on the availability of the energy source is difficult due to the unpredictability of energy sources. Routing protocol design is a challenge due to the fluctuation of the available energy in the network which affects which nodes will be awake at every point in time to receive broadcast packets. This makes broadcasting not suitable for WSN with energy harvesting [5]. The use of energy-aware duty cycling algorithms becomes a challenge if the energy on the nodes is dependent on the harvested energy. This could create erratic sleep/wake-up cycles since the residual energy may not be known a priori [121].

Inductive Coupling. Inductive coupling has been revised as a technique for wireless energy transfer to handle its limitation issues such as alignment and distance that are critical to their deployment and implementation. A strongly coupled inductive resonance coupling technique is introduced by [56] and has become a viable means of energy transfer. The challenge is the health implications of inductive resonance in the human environment due to constant exposure to radiations and the discomfort when used in humans [122]. Inductive coupling works within few centimeters, and therefore the distance of operation is limited. Scalability of transmission using inductive coupling is still a challenge since nodes must be tuned to avoid interference due to mutual coupling effect [123]. Since inductive coupling requires alignment of nodes, it is a challenge to design nodes with mobility.

6.2. Future Research Directions. Current research on modeling energy transfer is showing positive directions for single hop and multihop energy transfer. There is the need to 
develop or improve existing simulation tools to support energy transfer.

Single Energy Transmitters. The possibility of single energy transmitters transferring energy to multiple receivers simultaneously with multihop energy transfer with minimum charge and discharge losses is still open for research. Current research proposes using multiple transmitters to simultaneously charge nodes in large networks. But due to problem of signal interference with multiple chargers, there is a limit to the number that could be used in a network. The possibility of using a single energy transmitter that continuously receives energy through energy transfer could be a huge solution if multihop energy transfer is explored.

Extending Recent Simulation and Emulation Tools. Current simulation and emulation tools on the market (both commercial and open source) lack the full capability to test and evaluate new energy harvesting and transfer applications and protocols in WSN. For example, the current simulators and emulators used for modeling WSN applications such as Network Simulator 3 (NS-3) [88], Castalia Simulator [85], EKHO [66], OMNeT++ [86], COOJA [87], and PASES [67] do not reflect the network behavior accurately. Most of these simulators do not include models for energy transfer which makes performance evaluation difficult. Network Simulator three (NS-3) was recently extended to include two energy harvesting models [68]. The first model (i.e., the basic energy harvester) uses a generic random variable to provide energy to the harvester. The second model recharges the energy source with datasets based on real values obtained from solar panel. Development of simulation and emulation environments that support all aspects of energy harvesting and transfer is still an open research and will be a valuable tool to evaluate the proposed energy transfer techniques.

Experimental Demonstration of Multihop Energy Transfer. In WSN, the available energy harvested from the energy source may have practical implementation or simulation of multihop energy transfer that reduces the charge and discharge losses with better transfer efficiencies is still an open research. For charging nodes in an entire network, specialized devices such as mobile chargers or robots have been designed, where shortest path algorithms and optimal paths are developed for easy charging, but these specialized nodes increase the overall cost of energy transfer on test beds. The introduction of specialized nodes for energy harvesting and transfer called energy transmitters also increases the cost of implementation. Sensor nodes due to their usage and places of deployment are expected to be smaller in size; this becomes a challenge when antennas for energy transmission and reception must both be attached to ordinary nodes for receiving and transmitting energy. The need to design special nodes that could have contained both transmitter and receiver antennas on small sized nodes will enable the efficient implementation of energy transfer. There is also the need to develop optimal energy routing protocols to support multihop energy transfer that have the MAC and link layer support.

\section{Conclusions}

The role wireless sensor networks play in monitoring human activities in the last decade cannot be underestimated. Over the years, the introduction of energy management schemes that seek to prolong the lifetime of the sensor node and the overall network has been proposed, but the amount of energy required by the sensors to be operational all the time remains a challenge. In this paper, we have provided the trio energy management scheme that when fully implemented will keep the network alive forever. We first discussed the broad categorization of energy harvesting technologies and techniques and followed the discussion with the current energy transfer techniques and finally the approaches for conserving energy. Although there is an extensive work on each of these management schemes, there are still several other challenges that need to be addressed by the research community for effective implementation of the trio schemes.

\section{Conflicts of Interest}

The authors declare there are no conflicts of interest regarding this paper publication.

\section{References}

[1] S. Soro and W. B. Heinzelman, "Cluster head election techniques for coverage preservation in wireless sensor networks," Ad Hoc Networks, vol. 7, no. 5, pp. 955-972, 2009.

[2] C. Wang, J. Li, Y. Yang, and F. Ye, "Combining solar energy harvesting with wireless charging for hybrid wireless sensor networks," IEEE Transactions on Mobile Computing, vol. 17, no. 3, pp. 560-576, 2017.

[3] G. Anastasi, M. Conti, M. Di Francesco, and A. Passarella, "Energy conservation in wireless sensor networks: a survey," Ad Hoc Networks, vol. 7, no. 3, pp. 537-568, 2009.

[4] I. F. Akyildiz, W. Su, Y. Sankarasubramaniam, and E. Cayirci, "Wireless sensor networks: a survey," Computer Networks, vol. 38, no. 4, pp. 393-422, 2002.

[5] W. K. G. Seah, Z. A. Eu, and H.-P. Tan, "Wireless sensor networks powered by ambient energy harvesting (WSN-HEAP)survey and challenges," in Proceedings of the 1st International Conference on Wireless Communication, Vehicular Technology, Information Theory and Aerospace \& Electronic Systems Technology (Wireless VITAE '09), pp. 1-5, Aalborg, Denmark, May 2009.

[6] L. J. Chien, M. Drieberg, P. Sebastian, and L. H. Hiung, "A simple solar energy harvester for wireless sensor networks," in Proceedings of the 6 th International Conference on Intelligent and Advanced Systems (ICIAS '16), pp. 1-6, August 2016.

[7] S. Soro and W. B. Heinzelman, "Prolonging the lifetime of wireless sensor networks via unequal clustering," in Proceedings of the 19th IEEE International Parallel and Distributed Processing Symposium (IPDPS '05), pp. 236-243, Washington, DC, USA, April 2005.

[8] L. Xie, Y. Shi, Y. T. Hou, and A. Lou, "Wireless power transfer and applications to sensor networks," IEEE Wireless Communications Magazine, vol. 20, no. 4, pp. 140-145, 2013.

[9] T. Rault, A. Bouabdallah, and Y. Challal, "Energy efficiency in wireless sensor networks: a top-down survey," Computer Networks, vol. 67, pp. 104-122, 2014. 
[10] Y. Chen and Q. Zhao, "On the lifetime of wireless sensor networks," IEEE Communications Letters, vol. 9, no. 11, pp. 976978, 2005.

[11] I. Dietrich and F. Dressler, "On the lifetime of wireless sensor networks," ACM Transactions on Sensor Networks, vol. 5, no. 1, article 5, 2009.

[12] J. Ren, Y. Zhang, K. Zhang, A. Liu, J. Chen, and X. S. Shen, "Lifetime and Energy Hole Evolution Analysis in DataGathering Wireless Sensor Networks," IEEE Transactions on Industrial Informatics, vol. 12, no. 2, pp. 788-800, 2016.

[13] I. F. Akyildiz, W. Su, Y. Sankarasubramaniam, and E. Cayirci, "A survey on sensor networks," IEEE Communications Magazine, vol. 40, no. 8, pp. 102-105, 2002.

[14] T. Soyata, L. Copeland, and W. Heinzelman, "RF energy harvesting for embedded systems: a survey of tradeoffs and methodology," IEEE Circuits and Systems Magazine, vol. 16, no. 1, pp. 22-57, 2016.

[15] F. K. Shaikh and S. Zeadally, "Energy harvesting in wireless sensor networks: a comprehensive review," Renewable \& Sustainable Energy Reviews, vol. 55, pp. 1041-1054, 2016.

[16] M. Y. Naderi, K. R. Chowdhury, S. Basagni, W. Heinzelman, S. De, and S. Jana, "Experimental study of concurrent data and wireless energy transfer for sensor networks," in Proceedings of the IEEE Global Communications Conference (GLOBECOM '14), pp. 2543-2549, December 2014.

[17] R. J. M. Vullers, R. V. Schaijk, H. J. Visser, J. Penders, and C. Hoof, "Energy harvesting for autonomous wireless sensor networks," IEEE Journal of Solid-State Circuits, vol. 2, no. 2, pp. 29-38, 2010.

[18] R. Du, C. Fischione, and M. Xiao, "Joint node deployment and wireless energy transfer scheduling for immortal sensor networks," in Proceedings of the 15th International Symposium on Modeling and Optimization in Mobile, Ad Hoc, and Wireless Networks (WiOpt '17), pp. 1-8, Paris, France, May 2017.

[19] L. Xie, Y. Shi, Y. T. Hou, and H. D. Sherali, "Making sensor networks immortal: an energy-renewal approach with wireless power transfer," IEEE/ACM Transactions on Networking, vol. 20, no. 6, pp. 1748-1761, 2012.

[20] L. Xie, Y. Shi, Y. T. Hou, W. Lou, H. D. Sherali, and S. F. Midkiff, "On renewable sensor networks with wireless energy transfer: the multi-node case," in Proceedings of the 9th Annual IEEE Communications Society Conference on Sensor, Mesh and Ad Hoc Communications and Networks (SECON '12), pp. 10-18, June 2012.

[21] C. Zhu, K. Liu, C. Yu, R. Ma, and H. Cheng, "Simulation and experimental analysis on wireless energy transfer based on magnetic resonances," in Proceedings of the Vehicle Power and Propulsion Conference (VPPC '08), pp. 1-4, 2008.

[22] M. K. Watfa, H. AlHassanieh, and S. Selman, "Multi-hop wireless energy transfer in WSNs," IEEE Communications Letters, vol. 15, no. 12, pp. 1275-1277, 2011.

[23] A. A. Babayo, M. H. Anisi, and I. Ali, "A Review on energy management schemes in energy harvesting wireless sensor networks," Renewable \& Sustainable Energy Reviews, vol. 76, pp. 1176-1184, 2017.

[24] R. Doost-Mohammady and K. R. Chowdhury, "Transforming healthcare and medical telemetry through cognitive radio networks," IEEE Wireless Communications Magazine, vol. 19, no. 4, pp. 67-73, 2012.

[25] A. Khelil, F. K. Shaikh, P. Szczytowski, B. Ayari, and N. Suri, "Map-based design for autonomic wireless sensor networks," in Autonomic Communication, pp. 309-326, Springer, 2009.
[26] M. di Francesco, S. K. Das, and G. Anastasi, "Data collection in wireless sensor networks with mobile elements: a survey," ACM Transactions on Sensor Networks, vol. 8, no. 1, article 7, 2011.

[27] C. Alippi, G. Anastasi, M. Di Francesco, and M. Roveri, "Energy management in wireless sensor networks with energy-hungry sensors," IEEE Instrumentation \& Measurement Magazine, vol. 12, no. 2, pp. 16-23, 2009.

[28] M. A. Razzaque and S. Dobson, "Energy-efficient sensing in wireless sensor networks using compressed sensing," Sensors, vol. 14, no. 2, pp. 2822-2859, 2014.

[29] V. Raghunathan, S. Ganeriwal, and M. Srivastava, "Emerging techniques for long lived wireless sensor networks," IEEE Communications Magazine, vol. 44, no. 4, pp. 108-114, 2006.

[30] Y. C. Eldar and G. Kutyniok, Compressed Sensing: Theory and Applications, Cambridge University Press, 2012.

[31] E. Ever, R. Luchmun, L. Mostarda, A. Navarra, and P. Shah, "UHEED: an unequal clustering algorithm for wireless sensor networks," in Proceedings of the 1st International Conference on Sensor Networks (SENSORNETS '12), pp. 185-193, February 2012.

[32] A. Kansal, J. Hsu, S. Zahedi, and M. B. Srivastava, "Power management in energy harvesting sensor networks," $A C M$ Transactions on Embedded Computing Systems, vol. 6, no. 4, article 32, 2007.

[33] E. D. Dunlop, L. Wald, and M. Suri, Solar Energy Resource Management for Electricity Generation from Local Level to Global Scale, Nova Science Publishers Inc., 2006.

[34] F. Akhtar and M. H. Rehmani, "Energy replenishment using renewable and traditional energy resources for sustainable wireless sensor networks: a review," Renewable \& Sustainable Energy Reviews, vol. 45, pp. 769-784, 2015.

[35] P. T. V. Bhuvaneswari, R. Balakumar, V. Vaidehi, and P. Balamuralidhar, "Solar energy harvesting for wireless sensor networks," in Proceedings of the 1st International Conference on Computational Intelligence, Communication Systems and Networks (CICSYN '09), pp. 57-61, July 2009.

[36] P. Technologies, "Documentation," Powercast, Wireless Power for a Wireless World, 2018, http://www.powercastco.com/documentation/.

[37] K. S. Adu-Manu, N. Adam, C. Tapparello, H. Ayatollahi, and W. Heinzelman, "Energy-harvesting wireless sensor networks (EH-WSNs): a review," ACM Transactions on Sensor Networks (TOSN), vol. 14, no. 2, p. 10, 2018.

[38] T. J. Kazmierski and S. Beeby, Energy Harvesting Systems, Springer, 2014.

[39] C. Ó. Mathúna, T. O’Donnell, R. V. Martinez-Catala, J. Rohan, and B. O'Flynn, "Energy scavenging for long-term deployable wireless sensor networks," Talanta, vol. 75, no. 3, pp. 613-623, 2008.

[40] S. Roundy, P. K. Wright, and J. M. Rabaey, "Energy scavenging for wireless sensor networks," Norwell, 2003.

[41] D. M. Rowe, "Conversion efficiency and figure-of-merit," in CRC Handbook of Thermoelectrics, p. 31, CRC Press, 1995.

[42] I. Stark, "Invitedtalk: Thermal energy harvesting with thermo life," in Proceedings of the International Workshop Wearable and Implantable Body Sensor Networks (BSN '06), pp. 19-22, 2006.

[43] A. S. Aricò, P. Bruce, B. Scrosati, J.-M. Tarascon, and W. van Schalkwijk, "Nanostructured materials for advanced energy conversion and storage devices," Nature Materials, vol. 4, pp. 366-377, 2005. 
[44] F. I. Simjee and P. H. Chou, "Efficient charging of supercapacitors for extended lifetime of wireless sensor nodes," IEEE Transactions on Power Electronics, vol. 23, no. 3, pp. 1526-1536, 2008.

[45] T. H. Ng and W. H. Liao, "Sensitivity analysis and energy harvesting for a self-powered piezoelectric sensor," Journal of Intelligent Material Systems and Structures, vol. 16, no. 10, pp. 785-797, 2005.

[46] N. S. Shenck and J. A. Paradiso, "Energy scavenging with shoemounted piezoelectrics," IEEE Micro, vol. 21, no. 3, pp. 30-42, 2001.

[47] G. K. Ottman, H. F. Hofmann, A. C. Bhatt, and G. A. Lesieutre, "Adaptive piezoelectric energy harvesting circuit for wireless remote power supply," IEEE Transactions on Power Electronics, vol. 17, no. 5, pp. 669-676, 2002.

[48] H. A. Sodano, D. J. Inman, and G. Park, "Comparison of piezoelectric energy harvesting devices for recharging batteries," Journal of Intelligent Material Systems and Structures, vol. 16, no. 10, pp. 799-807, 2005.

[49] M. J. Guan and W. H. Liao, "Characteristics of energy storage devices in piezoelectric energy harvesting systems," Journal of Intelligent Material Systems and Structures, vol. 19, no. 6, pp. 671680, 2008.

[50] V. Raghunathan, A. Kansal, J. Hsu, J. Friedman, and M. Srivastava, "Design considerations for solar energy harvesting wireless embedded systems," in Proceedings of the 4th International Symposium on Information Processing in Sensor Networks (IPSN '05), pp. 457-462, April 2005.

[51] B. Buchli, F. Sutton, J. Beutel, and L. Thiele, "Dynamic power management for long-term energy neutral operation of solar energy harvesting systems," in Proceedings of the 12th ACM Conference, pp. 31-45, November 2014.

[52] S. Peng, T. Wang, and C. P. Low, "Energy neutral clustering for energy harvesting wireless sensors networks," Ad Hoc Networks, vol. 28, pp. 1-16, 2015.

[53] S. Baghaee, S. Chamanian, H. Ulusan, O. Zorlu, E. UysalBiyikoglu, and H. Kulah, "Demonstration of energy-neutral operation on a WSN testbed using vibration energy harvesting," in Proceedings of the 20th European Wireless Conference (EW '14), pp. 47-52, May 2014.

[54] C. M. Vigorito, D. Ganesan, and A. G. Barto, "Adaptive control of duty cycling in energy-harvesting wireless sensor networks," in Proceedings of the 4th Annual IEEE Communications Society Conference on Sensor, Mesh and Ad Hoc Communications and Networks (SECON '07), pp. 21-30, IEEE, San Diego, Calif, USA, June 2007.

[55] Y. Shi, L. Xie, Y. T. Hou, and H. D. Sherali, "On renewable sensor networks with wireless energy transfer," in Proceedings of the IEEE INFOCOM '11, pp. 1350-1358, Shanghai, China, April 2011.

[56] A. Kurs, A. Karalis, R. Moffatt, J. D. Joannopoulos, P. Fisher, and M. Soljacic, "Wireless power transfer via strongly coupled magnetic resonances," Science, vol. 317, no. 5834, pp. 83-86, 2007.

[57] X. Wei, Z. Wang, and H. Dai, "A critical review of wireless power transfer via strongly coupled magnetic resonances," Energies, vol. 7, no. 7, pp. 4316-4341, 2014.

[58] F. Musavi and W. Eberle, "Overview of wireless power transfer technologies for electric vehicle battery charging," IET Power Electronics, vol. 7, no. 1, pp. 60-66, 2014.

[59] O. Jonah and S. V. Georgakopoulos, "Wireless power transfer in concrete via strongly coupled magnetic resonance," IEEE
Transactions on Antennas and Propagation, vol. 61, no. 3, pp. 1378-1384, 2013.

[60] F. Engmann, J.-D. Abdulai, and J. Q. Azasoo, "Enhancing the reliability of wsn through wireless energy transfer," in Proceedings of the International Conference on Computational Science and Its Applications, pp. 610-618, Springer, 2016.

[61] J. Li, K. Li, and W. Zhu, "Improving sensing coverage of wireless sensor networks by employing mobile robots," in Proceedings of the IEEE International Conference on Robotics and Biomimetics (ROBIO '07), pp. 899-903, December 2007.

[62] B. L. Cannon and D. D. Stancil, "Magnetic resonant coupling as a potential means for wireless power transfer to multiple small receivers," IEEE Transactions on Power Electronics, vol. 24, no. 7, pp. 1819-1825, 2009.

[63] M. Erol-Kantarci and H. T. Mouftah, "Suresense: sustainable wireless rechargeable sensor networks for the smart grid," IEEE Wireless Communications Magazine, vol. 19, no. 3, pp. 30-36, 2012.

[64] K. S. Adu-Manu, C. Tapparello, W. Heinzelman, F. A. Katsriku, and J.-D. Abdulai, "Water quality monitoring using wireless sensor networks: Current trends and future research directions," ACM Transactions on Sensor Networks, vol. 13, no. 1, 2017.

[65] D. Mishra, S. De, S. Jana, S. Basagni, K. Chowdhury, and W. Heinzelman, "Smart RF energy harvesting communications: Challenges and opportunities," IEEE Communications Magazine, vol. 53, no. 4, pp. 70-78, 2015.

[66] J. Hester, T. Scott, and J. Sorber, "Ekho: realistic and repeatable experimentation for tiny energy-harvesting sensors," in Proceedings of the 12th ACM Conference on Embedded Networked Sensor Systems (SenSys '14), pp. 1-15, New York, NY, USA, November 2014.

[67] I. Minakov and R. Passerone, "PASES: an energy-aware design space exploration framework for wireless sensor networks," Journal of Systems Architecture, vol. 59, no. 8, pp. 626-642, 2013.

[68] C. Tapparello, H. Ayatollahi, and W. Heinzelman, "Energy harvesting framework for network simulator 3 (ns-3)," in Proceedings of the 2nd International Workshop on Energy Neutral Sensing Systems (ENSsys '14), pp. 37-42, Memphis, TN, USA, November 2014.

[69] H. P. Dai, X. B. Wu, L. J. Xu, G. Chen, and S. Lin, "Using minimum mobile chargers to keep large-scale wireless rechargeable sensor networks running forever," in Proceedings of the IEEE 22nd International Conference on Computer Communication and Networks (ICCCN '13), pp. 1-7, August 2013.

[70] W. Xu, W. Liang, X. Lin, and G. Mao, "Efficient scheduling of multiple mobile chargers for wireless sensor networks," IEEE Transactions on Vehicular Technology, vol. 65, no. 9, pp. 76707683, 2016.

[71] Y. Peng, Z. Li, W. Zhang, and D. Qiao, "Prolonging sensor network lifetime through wireless charging," in Proceedings of the 31st IEEE Real-Time Systems Symposium (RTSS '10), pp. 129139, IEEE, December 2010.

[72] W. Yao, M. Li, and M.-Y. Wu, "Inductive charging with multiple charger nodes in wireless sensor networks," in Proceedings of the Asia-Pacific Web Conference, pp. 262-270, Springer, 2006.

[73] A. Madhja, S. Nikoletseas, and T. P. Raptis, "Hierarchical, collaborative wireless energy transfer in sensor networks with multiple Mobile Chargers," Computer Networks, vol. 97, pp. 98$112,2016$.

[74] H. Ayatollahi, C. Tapparello, and W. Heinzelman, "Transmitterreceiver energy efficiency: a trade-off in MIMO wireless sensor 
networks," in Proceedings of the IEEE Wireless Communications and Networking Conference (WCNC '15), pp. 1476-1481, March 2015.

[75] A. B. Constantine, "Microstrip Antennas," in Antenna Theory: Analysis and Design, John Wiley \& Sons, 3rd edition, 2005.

[76] Q. Li, R. Q. Hu, Y. Qian, and G. Wu, "Cooperative communications for wireless networks: techniques and applications in LTEadvanced systems," IEEE Wireless Communications Magazine, vol. 19, no. 2, pp. 22-29, 2012.

[77] K. Huang and E. Larsson, "Simultaneous information and power transfer for broadband wireless systems," IEEE Transactions on Signal Processing, vol. 61, no. 23, pp. 5972-5986, 2013.

[78] A. A. Nasir, X. Zhou, S. Durrani, and R. A. Kennedy, "Relaying protocols for wireless energy harvesting and information processing," IEEE Transactions on Wireless Communications, vol. 12, no. 7, pp. 3622-3636, 2013.

[79] R. Zhang and C. K. Ho, "MIMO broadcasting for simultaneous wireless information and power transfer," IEEE Transactions on Wireless Communications, vol. 12, no. 5, pp. 1989-2001, 2013.

[80] J. Park and B. Clerckx, "Joint wireless information and energy transfer with reduced feedback in MIMO interference channels," IEEE Journal on Selected Areas in Communications, vol. 33, no. 8, pp. 1563-1577, 2015.

[81] Z. Xiang and M. Tao, "Robust beamforming for wireless information and power transmission," IEEE Wireless Communications Letters, vol. 1, no. 4, pp. 372-375, 2012.

[82] X. Chen, C. Yuen, and Z. Zhang, "Wireless energy and information transfer tradeoff for limited-feedback multiantenna systems with energy beamforming," IEEE Transactions on Vehicular Technology, vol. 63, no. 1, pp. 407-412, 2014.

[83] J. Park and B. Clerckx, "Joint wireless information and energy transfer in a two-user MIMO interference channel," IEEE Transactions on Wireless Communications, vol. 12, no. 8, pp. 4210-4221, 2013.

[84] J. Park and B. Clerckx, "Joint wireless information and energy transfer in a K-user MIMO interference channel," IEEE Transactions on Wireless Communications, vol. 13, no. 10, pp. 5781-5796, 2014.

[85] Castalia, "Castalia wireless sensor network simulator," 2016.

[86] F. Chen, I. Dietrich, R. German, and F. Dressler, "An energy model for simulation studies of wireless sensor networks using OMNeT++," Praxis der Informationsverarbeitung und Kommunikation, vol. 32, no. 2, pp. 133-138, 2009.

[87] H. Sundani, H. Li, V. Devabhaktuni, M. Alam, and P. Bhattacharya, "Wireless sensor network simulators a survey and comparisons," International Journal of Computer Networks, vol. 2, no. 5, pp. 249-265, 2011.

[88] H. Wu, S. Nabar, and R. Poovendran, "An energy framework for the network simulator 3 (ns-3)," in Proceedings of the 4th International ICST Conference on Simulation Tools and Techniques, Barcelona, Spain, March 2011.

[89] M. K. Stojcev, M. R. Kosanovic, and L. R. Golubovic, "Power management and energy harvesting techniques for wireless sensor nodes," in Proceedings of the 9th International Conference on Telecommunications in Modern Satellite, Cable, and Broadcasting Services (TELSIKS '09 ), pp. 65-72, October 2009.

[90] S. Cui, A. J. Goldsmith, and A. Bahai, "Energy-efficiency of MIMO and cooperative MIMO techniques in sensor networks," IEEE Journal on Selected Areas in Communications, vol. 22, no. 6, pp. 1089-1098, 2004.
[91] S. Hussain, A. Azim, and J. H. Park, "Energy efficient virtual MIMO communication for wireless sensor networks," Telecommunication Systems, vol. 42, no. 1-2, pp. 139-149, 2009.

[92] L. Lu, G. Y. Li, A. L. Swindlehurst, A. Ashikhmin, and R. Zhang, "An overview of massive MIMO: benefits and challenges," IEEE Journal of Selected Topics in Signal Processing, vol. 8, no. 5, pp. 742-758, 2014.

[93] A. Nosratinia, T. E. Hunter, and A. Hedayat, "Cooperative communication in wireless networks," IEEE Communications Magazine, vol. 42, no. 10, pp. 74-80, 2004.

[94] S. Basagni, M. Y. Naderi, C. Petrioli, and D. Spenza, "Wireless sensor networks with energy harvesting," Mobile Ad Hoc Networking: Cutting Edge Directions, pp. 701-736, 2013.

[95] I. Khemapech, A. Miller, and I. Duncan, "A survey of transmission power control in wireless sensor networks," in Proceedings of the PGNet, pp. 15-20, 2007.

[96] S. Lin, F. Miao, J. Zhang et al., "ATPC: Adaptive transmission power control for wireless sensor networks," ACM Transactions on Sensor Networks, vol. 12, no. 1, 2016.

[97] K. V. Kumar and E. Baburaj, "Energy efficient transmission power control in sensor nodes of WSN SCADA systems using cognitive fuzzy systems," International Journal of Applied Engineering Research, vol. 11, no. 2, pp. 1478-1484, 2016.

[98] H. Cotuk, K. Bicakci, B. Tavli, and E. Uzun, "The impact of transmission power control strategies on lifetime of wireless sensor networks," IEEE Transactions on Computers, vol. 63, no. 11, pp. 2866-2879, 2014.

[99] A. Tripathi, S. Gupta, and B. Chourasiya, "Survey on data aggregation techniques for wireless sensor networks," International Journal of Advanced Research in Computer and Communication Engineering, vol. 3, no. 7, pp. 7366-7371, 2014.

[100] L. C. Zhong, R. Shah, C. Guo, and J. Rabaey, "An ultra-low power and distributed access protocol for broadband wireless sensor networks," IEEE Broadband Wireless Summit, vol. 3, 2001.

[101] M. Bagaa, Y. Challal, A. Ksentini, A. Derhab, and N. Badache, "Data aggregation scheduling algorithms in wireless sensor networks: solutions and challenges," IEEE Communications Surveys \& Tutorials, vol. 16, no. 3, pp. 1339-1368, 2014.

[102] N. Kimura and S. Latifi, "A survey on data compression in wireless sensor networks," in Proceedings of the International Conference on Information Technology: Coding and Computing (ITCC '05), vol. 2, pp. 8-13, April 2005.

[103] T. Srisooksai, K. Keamarungsi, P. Lamsrichan, and K. Araki, "Practical data compression in wireless sensor networks: a survey," Journal of Network and Computer Applications, vol. 35, no. 1, pp. 37-59, 2012.

[104] G. M. Dias, B. Bellalta, and S. Oechsner, "A survey about prediction-based data reduction in wireless sensor networks," ACM Computing Surveys, vol. 49, no. 3, 2016.

[105] C. Alippi, G. Anastasi, M. Di Francesco, and M. Roveri, "An adaptive sampling algorithm for effective energy management in wireless sensor networks with energy-hungry sensors," IEEE Transactions on Instrumentation and Measurement, vol. 59, no. 2, pp. 335-344, 2010.

[106] B. Srbinovski, M. Magno, B. O’Flynn, V. Pakrashi, and E. Popovici, "Energy aware adaptive sampling algorithm for energy harvesting wireless sensor networks," in Proceedings of the Sensors Applications Symposium (SAS '15), pp. 1-6, 2015.

[107] Y. Yin, F. Liu, X. Zhou, and Q. Li, "An efficient data compression model based on spatial clustering and principal component analysis in wireless sensor networks," Sensors, vol. 15, no. 8, pp. 19443-19465, 2015. 
[108] X. Cao, S. Madria, and T. Hara, "Efficient Z-order encoding based multi-modal data compression in WSNs," in Proceedings of the 37th IEEE International Conference on Distributed Computing Systems (ICDCS '17), pp. 2185-2192, June 2017.

[109] M. Wu, L. Tan, and N. Xiong, "Data prediction, compression, and recovery in clustered wireless sensor networks for environmental monitoring applications," Information Sciences, vol. 329, pp. 800-818, 2016.

[110] B. Stojkoska, D. Solev, and D. Davcev, "Data prediction in WSN using variable step size LMS algorithm," in Proceedings of the 5th International Conference on Sensor Technologies and Applications (SENSORCOMM '11), pp. 191-196, August 2011.

[111] W. R. Heinzelman, A. Chandrakasan, and H. Balakrishnan, "Energy-efficient communication protocol for wireless microsensor networks," in Proceedings of the 33rd Annual Hawaii International Conference on System Siences (HICSS '00), p. 10, January 2000.

[112] M. Ye, C. Li, G. Chen, and J. Wu, "EECS: an energy efficient clustering scheme in wireless sensor networks," in Proceedings of the 24th IEEE International Performance, Computing, and Communications Conference (IPCCC '05), pp. 535-540, IEEE, April 2005.

[113] G. V. Selvi and R. Manoharan, "A survey of energy efficient unequal clustering algorithms for wireless sensor networks," International Journal of Computer Applications, vol. 79, no. 1, 2013.

[114] R. Sharma, G. Jain, and S. Gupta, "Enhanced Cluster-head selection using round robin technique in WSN," in Proceedings of the International Conference on Communication Networks (ICCN '15), pp. 37-42, Gwalior, India, November 2015.

[115] A. A. Abbasi and M. Younis, "A survey on clustering algorithms for wireless sensor networks," Computer Communications, vol. 30, no. 14-15, pp. 2826-2841, 2007.

[116] C. Alippi, R. Camplani, C. Galperti, and M. Roveri, "A robust, adaptive, solar-powered WSN framework for aquatic environmental monitoring," IEEE Sensors Journal, vol. 11, no. 1, pp. 4555, 2011.

[117] G. Chen, C. Li, M. Ye, and J. Wu, "An unequal clusterbased routing protocol in wireless sensor networks," Wireless Networks, vol. 15, no. 2, pp. 193-207, 2009.

[118] H. Bagci and A. Yazici, "An energy aware fuzzy approach to unequal clustering in wireless sensor networks," Applied Soft Computing, vol. 13, no. 4, pp. 1741-1749, 2013.

[119] K. Kaushik, D. Mishra, S. De et al., "Experimental demonstration of multi-hop RF energy transfer," in Proceedings of the IEEE 24th Annual International Symposium on Personal, Indoor, and Mobile Radio Communications (PIMRC '13), pp. 538-542, September 2013.

[120] G. Benigno, O. Briante, and G. Ruggeri, "A sun energy harvester model for the network simulator 3 (ns-3)," in Proceedings of the 12th Annual IEEE International Conference on Sensing, Communication, and Networking - Workshops (SECON Workshops '15), pp. 1-6, Seattle, WA, USA, June 2015.

[121] R. C. Carrano, D. Passos, L. C. S. Magalhaes, and C. V. N. Albuquerque, "Survey and taxonomy of duty cycling mechanisms in wireless sensor networks," IEEE Communications Surveys \& Tutorials, vol. 16, no. 1, pp. 181-194, 2014.

[122] I. Mayordomo, T. Drager, P. Spies, J. Bernhard, and A. Pflaum, "An overview of technical challenges and advances of inductive wireless power transmission," in Proceedings of the IEEE, vol. 101, no. 6, pp. 1302-1311, 2013.
[123] S. Bi, C. K. Ho, and R. Zhang, "Wireless powered communication: opportunities and challenges," IEEE Communications Magazine, vol. 53, no. 4, pp. 117-125, 2015. 


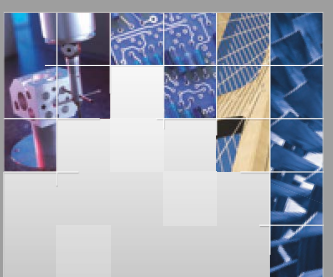

\section{Enfincering}
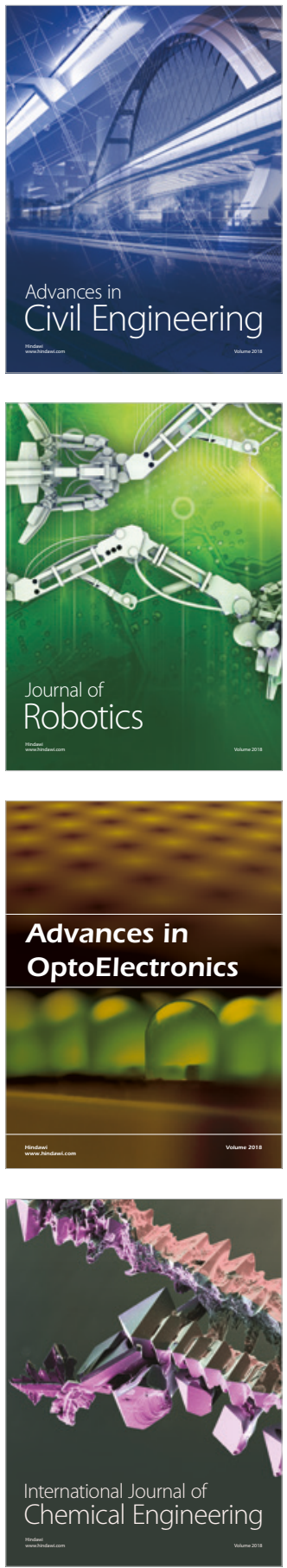

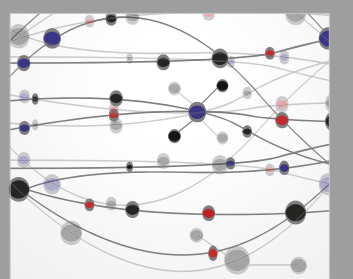

\section{Rotating \\ Machinery}

The Scientific World Journal

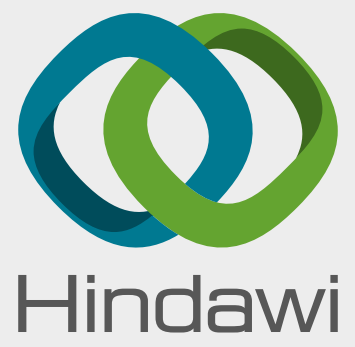

Submit your manuscripts at

www.hindawi.com
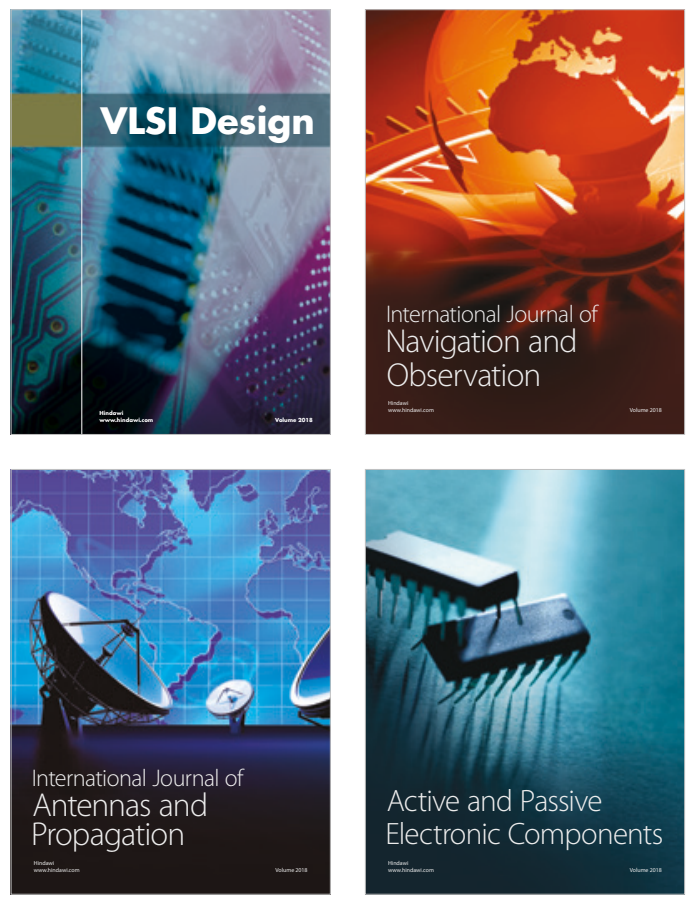
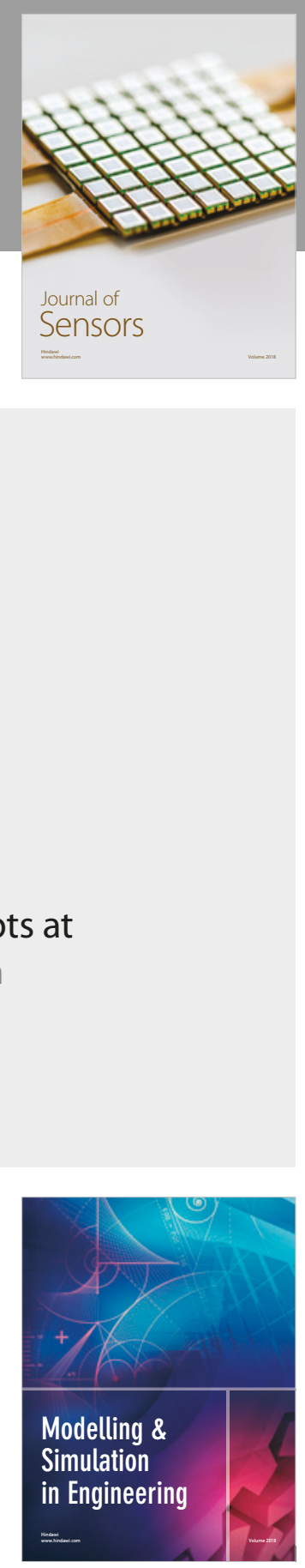

\section{Advances \\ Multimedia}
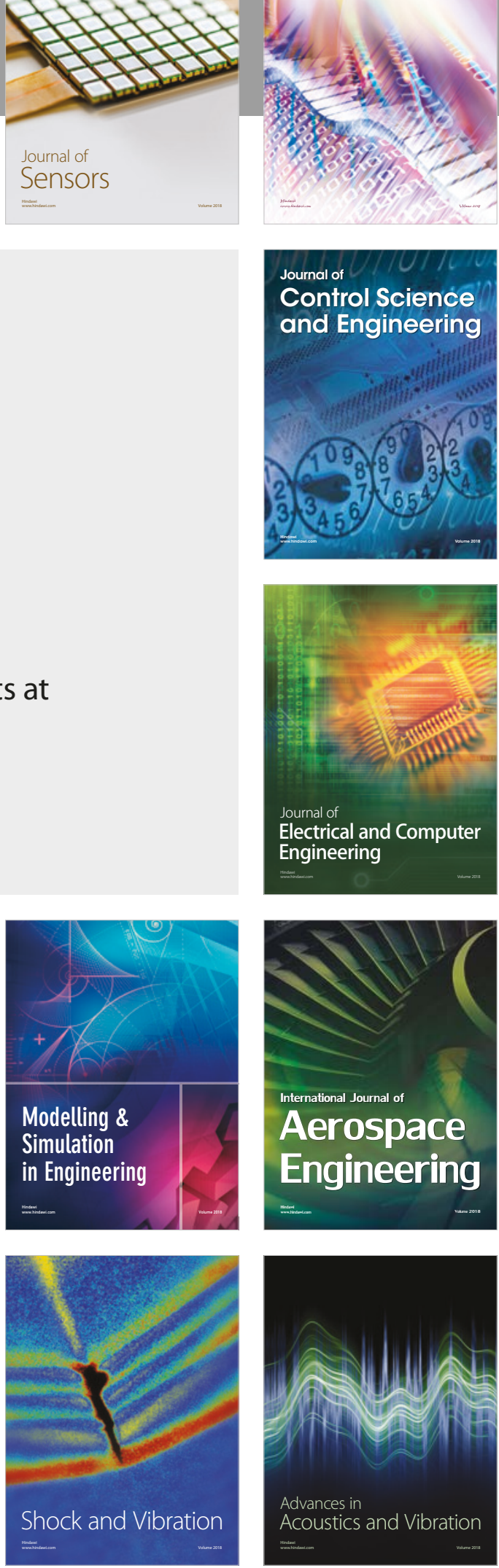